\title{
Confiance et Technologie : Deux Dimensions de l'Innovation Ouverte et Agile appliquée à l'Énergie
}

\author{
Confidence and Technology: Two Dimensions to Open and Agile \\ Innovation Applied to Energy
}

\author{
Laurent Dupont ${ }^{1}$, Joëlle Mastelic ${ }^{2}$, Nathalie Nyffeler ${ }^{2}$, Sophie Latrille ${ }^{2}$, Éric Seulliet ${ }^{3}$ \\ ${ }^{1}$ Université de Lorraine, Laboratoire ERPI, France, I.dupont@univ-lorraine.fr \\ ${ }^{2}$ Haute École Spécialisée de Suisse Occidentale (HES-S0), Suisse, \{joelle.mastelic ; sophie.latrille\}@hevs.ch ; \\ nathalie.nyffeler@heig-vd.ch \\ ${ }^{3}$ La Fabrique du Futur, France, eric@lafabriquedufutur.org
}

\begin{abstract}
RÉSUMÉ. L'Innovation Ouverte est un champ de recherche largement exploré et de nombreux dispositifs technologiques sont développés pour soutenir l'implication des usagers, ou autres acteurs extérieurs d'une entreprise, dans son processus de co-création distribuée, i.e. lorsque les acteurs travaillent de manière asynchrone et à distance géographique. Un des paramètres fondamentaux au succès de démarches collaboratives distribuées est la confiance que les acteurs s'accordent entre eux, dans le processus en cours et aux technologies auxquels ils sont confrontés. La notion de confiance reste néanmoins encore peu utilisée comme paramètre de pilotage et de soutien au déploiement de projet de co-création. Le manque de compréhension des mécanismes en jeu semble expliquer cette situation. A travers l'analyse de deux études de cas de co-création appliquée au domaine de l'énergie, cet article propose d'identifier les leviers, notamment technologiques, favorisant la confiance entre les parties et par extension l'agilité du processus. En complément d'un éclairage des pratiques, cet article fournit un premier cadre de pilotage de projet de co-création pour les praticiens à travers la conception du modèle « co-con ».

ABSTRACT. Open Innovation is a widely explored field of research and many technologies have been developed to support the involvement of users, or other external stakeholders of a company, in its distributed co-creation process, i.e. when actors work asynchronously and at geographical distance. One of the fundamental parameters to the success of distributed collaborative approaches is the trust that the actors have in each other, in the current process and in technology. However, the notion of trust is still rarely used as a parameter for piloting and supporting co-creation project deployment. The lack of understanding of the mechanisms involved seems to explain this situation. Using the analysis of two case studies of co-creation in the field of energy, this paper proposes to identify the levers, notably technological ones, favoring the confidence between stakeholders and by extension the agility of the process. In addition to illuminating these practices, this paper provides a first co-creation project management framework for practitioners, through the design of the "co-con" model.

MOTS-CLÉS. Confiance, Innovation ouverte, Innovation agile, Living lab, plateforme de co-création.

KEYWORDS. Open Innovation, Agile Innovation, Living Lab, Co-creative Platform.
\end{abstract}

\section{Introduction - L'enjeu de I'Innovation Ouverte et Agile}

Chesbrough [CHESBROUGH, 03] a introduit et défini le concept d'Innovation Ouverte (IO) : «Open Innovation means that valuable ideas can come from inside or outside the company and can go to market from inside or outside the company as well. » Cette notion et ses pratiques liées sont à présent largement répandues dans le monde socio-économique. Dans ce contexte, l'enjeu n'est plus tant de diffuser l'IO mais d'identifier les leviers pour la renforcer en s'appuyant notamment sur les domaines liés à ce concept phare. Parmi les axes de développement de l'IO nous pouvons notamment citer l'innovation par l'usage que nous aborderons par le biais des Living Labs (LL) et l'Innovation Agile (IA). Bien que popularisé par la notion d'agilité dans le domaine informatique [HIGHSMITH, FOWLER, 01], l'IA se décline à différentes échelles, partant de l'équipe restreinte au sein d'une entreprise aux organisations complètes qu'elles soient industrielles ou gouvernementales. Par exemple, [DIKERT et al., 16] étudient notamment les transformations agiles au niveau des grandes échelles industrielles. [MERGEL, 16] concentre ses travaux sur les gouvernements. 
$\mathrm{Au}$ sein d'une organisation, l'innovation agile sous-tend un double processus d'ouverture. Le premier niveau d'ouverture concerne les équipes de projet en phase amont de l'innovation, dont il est attendu que les membres échangent régulièrement avec le client final, voire l'usager du produit ou service qui est en phase de conception. Par ailleurs, l'organisation elle-même doit s'inscrire dans une démarche d'innovation ouverte nécessitant l'interaction entre les équipes, et tout ou partie de l'organisation avec son écosystème (fournisseurs, partenaires économiques, institutionnels, académiques, etc.) Ainsi, la reconnaissance accrue de l'inventivité et de l'innovation comme facteurs différentiateurs indispensables dans un monde de plus en plus concurrentiel, l'essor de l'économie collaborative qui implique des échanges soutenus entre pairs et l'intérêt marqué pour introduire un principe de co-création dans des projets d'innovation renforce, diffuse, voire invite à repenser l'IO, les relations interpersonnelles et la gouvernance dans un collectif.

Dans un tel contexte, afin que le système fonctionne et se développe de façon optimale et fluide, il est utile d'identifier ce qui peut enrayer ou - au contraire - stimuler la motivation des individus à s'engager comme co-créateurs ou au moins contributeur d'un processus sur lequel il semble n'avoir aucune emprise.

Parmi les obstacles des démarches d'IO, on retrouve entre autres le risque qu'un contributeur survalorise ses propres apports et minimise ceux de ses pairs. Méfiance, jalousie, dénigrement pouvant se cumuler à ce manque de discernement provoquent ainsi de la rétention d'information et de la réticence à coopérer. La difficulté à mesurer, donc à valoriser les diverses contributions et leurs impacts est un autre frein. Les solutions juridiques actuelles (brevets, NDA, enveloppes Soleau, etc.) n'offrent pas de réponse satisfaisante, notamment dans le cas de contributeurs multiples membres d'organisation différentes, en particulier pour des projets de grande envergure ou répondant à des enjeux de société. Ces situations peuvent générer de l'inertie et des déperditions amenant a priori un manque d'efficacité global. Un certain nombre de questions restent en suspens pour qualifier plus précisément l'efficacité et la performance du système d'innovation considéré. Comment identifier, protéger et répartir la valeur créée lorsque les contributeurs appartiennent à des organisations différentes, voire lorsque certains des contributeurs s'engagent à titre personnel alors que d'autres le font dans le cadre d'un emploi ou d'une fonction ? Les acteurs mobilisés font-ils référence aux mêmes valeurs ? Est-il nécessaire d'adhérer aux mêmes valeurs pour contribuer à un processus collectif ? Le cas échéant, comment identifier précisément le niveau de contribution de différents participants ? Comment évaluer, voire mesurer, en quoi les contributions des uns et des autres résonnent entre elles, générant parfois une idée exceptionnelle de la part d'une seule personne ? Ne conviendrait-il d'ailleurs pas de dépasser la question de la reconnaissance individuelle dans un processus considéré comme pleinement collectif et collaboratif ? Quel serait l'environnement propice à l'IO, propre à stimuler les initiatives individuelles mais aussi le partage et la collaboration nourries des intelligences collectives?

Nous ne pourrons pas traiter l'ensemble de ces questions, qui ne sont d'ailleurs sans doute pas exhaustives. Ce papier se concentre sur les liens entre confiance, technologie et co-création. En effet, les recherches montrent que la confiance est un facteur clé de succès dans le développement des organisations et l'intégration des usagers dans les processus d'innovation ouverte comme nous le verrons dans la section suivante. Par ailleurs, [GRECO et al., 17] soulignent les apports potentiels de l'IO pour le secteur de l'énergie avec le rôle clé de cinq variables : l'implication du gouvernement, la participation des universités, l'implication des clients et des fournisseurs, la capacité d'absorption et la nouveauté de l'innovation. Plus spécifiquement, dans l'univers de la distribution d'énergie, la cocréation représente une opportunité pour le développement de la relation clients et de la création de valeur (par exemple, la conception de nouvelle technologie ou de nouveaux services). Le défi est de mettre l'usager au centre de l'innovation énergétique, au début de la chaîne de création de valeur, dans des métiers et pratiques plutôt tournés vers l'innovation technologique [DUPONT et al., 17]. Cette évolution du contexte offre l'opportunité de comprendre, voire mesurer, au plus tôt dans l'arrivée de nouvelles pratiques, l'apport de l'intégration des usagers au processus d'innovation dans le domaine des services énergétiques. Ainsi, à travers deux cas d'étude liés à l'énergie, nous illustrerons en quoi la (C) 2018 ISTE OpenScience - Published by ISTE Ltd. London, UK - openscience.fr

Page $\mid 2$ 
confiance est un enjeu majeur, particulièrement dans les situations où les projets reposent sur une recherche d'une large diversité des acteurs en quantité comme en qualité (i.e. champs de compétences, centre d'intérêt.) Enfin, nous proposerons de mettre en exergue et d'articuler les technologies favorisant la mobilisation et l'adhésion des parties prenantes au processus de co-création. Nous parlons dès lors des technologies en lien avec les démarches et organisations collaboratives. Ces dernières regroupent des outils, des méthodes, des systèmes numériques (du Web 2.0 aux dispositifs immersifs), voire des espaces physiques qui permettent et facilitent le travail de groupes composés de personnes éclectiques, distribuées ou non, en travail synchrone ou asynchrone [BAYRAK, 15, DUPONT ET AL., 18, RECKER ET AL., 13, TRAN, 14]. Nous cherchons ainsi à identifier les fonctionnalités « idéales » que devrait remplir une technologie ou plusieurs technologies complémentaires pour accompagner la cocréation. Le cas échéant, nous cherchons également à voir dans quel cas la technologie facilite, ou au contraire freine l'émergence et l'appropriation des idées. Le challenge étant de vérifié qu'une idée est appropriée ou rejetée pour ce quelle est, et non pas à cause de la technologique qui sert à la faire émerger et à la représenter ou à cause de l'acteur initial qui la porte. A travers, l'esquisse d'un cahier des charges fonctionnel, nous soulignerons plus spécifiquement les caractéristiques que doit offrir un dispositif technologique intégré ou un ensemble de technologies renforçant l'Innovation Ouverte et Agile.

\section{L'enjeu de la confiance pour l'innovation}

\subsection{La confiance : un levier essentiel pour les innovations inter et intra entreprises}

La performance d'une démarche d'IO peut être renforcée en réduisant, par exemple, le temps d'accès au marché pour les nouveaux produits ou services. Travailler sur la robustesse du processus peut être bénéfique, en s'assurant par exemple d'être plus en phase avec les besoins des utilisateurs. L'IO peut également devenir plus écoresponsable en mobilisant des processus respectueux des principes de soutenabilité en privilégiant des circuits courts, des choix socialement responsables, le bien-être de salariés, etc. Un des dénominateurs communs à l'ensemble de ces axes d'amélioration est la confiance. Celle-ci se retrouve à tous les niveaux d'une organisation et peut être un levier de développement: confiance entre pairs dans une communauté de co-créateurs, confiance entre entreprises et contributeurs externes, confiance entre une entreprise et ses collaborateurs, confiance entre partenaires, confiance dans les méthodes et outils sous-tendant l'IO.

La confiance contient au moins deux dimensions [MAYER et al., 95] : la bienveillance (de l'autre à laquelle on se soumet) et la capacité (de l'autre à disposer des compétences, savoirs, savoir-faire dans un domaine donné.) L'intégrité peut également être considérée comme une des composantes de la confiance [FAWCETT et al., 12]. Ici, l'intégrité correspond à l'adhésion à un groupe de valeurs comme le respect de ses engagements. Reprenant ces principes on constate que plusieurs niveaux de maturité en termes de confiance peuvent être observés et développés au sein des entreprises et de leurs relations partenariales. Ainsi, des chercheurs proposent un modèle qui va de la «confiance limitée » à la « confiance collaborative ».

Au sein des entreprises, les travaux de [PIROLA-MERLO, 10], reprenant le modèle « innovative team climate » de West, montrent qu'il semble y avoir une corrélation positive entre le climat d'une équipe et la rapidité à achever un projet de recherche et développement. Quatre dimensions caractérisent ce climat [WEST, 90] : (1) le partage d'objectifs clairs et appréciés ; (2) un environnement non menaçant où les membres peuvent influencer les discussions et les décisions ; (3) le souci d'atteindre l'excellence grâce à un travail de qualité et à une évaluation critique ; (4) la valorisation de l'innovation et le soutien de pratiques de travail visant à réaliser l'innovation. [WEST, SACRAMENTO, 06] rappellent que certains auteurs considèrent que l'apprentissage et l'innovation ne peuvent avoir lieu que lorsque les membres du groupe font confiance aux intentions des autres membres. 
Des travaux de 2014, menés sur un panel de 48 entreprises membres de l'association allemande des services de maintenance, montrent que trois éléments sont nécessaires pour obtenir un niveau d'innovation de moyen à fort : contrats, confiance envers un fournisseur à respecter la collaboration et capacité à remplir les obligations convenues entre parties [VAN DER VALK et al., 16]. Ces mêmes travaux montrent que pour atteindre certains niveaux d'innovation alors certains seuils doivent être respectés pour chacun de ces trois paramètres. Dans certaines conditions, la performance d'innovation dépend très directement du niveau de détail des contrats et de la qualité de la confiance établie entre les partenaires. Au niveau des entreprises la mise en place et le maintien de relations B-to-B basées sur la confiance réduisent les risques, les coûts de transaction et les relations à long terme [DOVEY, 09]. Ce point est particulièrement vrai au niveau des processus d'innovation et de créativité [SHAZI et al., 15].

L'innovation demande de la créativité et une certaine prise de risque, les résultats sont dès lors incertains et imprédictibles rendant impossible la rédaction à l'avance des termes et clauses précis d'un contrat. Des mécanismes plus souples et flexibles sont nécessaires pour encadrer un partenariat ou une collaboration, dans lesquels il faut savoir jongler entre la confiance entre les partenaires et la mise en place de contrats. Par ailleurs, une enquête de 2011 [WANG et al., 11] auprès de 315 entreprises chinoises montre que si, dans certaines circonstances, contrat et confiance peuvent se substituer et garantir la même performance, dans les situations d'incertitude la confiance entre les parties est bien plus efficace que la mise en place (couteuse) de contrats. Gérer un projet d'IO nécessite d'avoir des capacités à comprendre et anticiper l'environnement dans lequel va se déployer le projet.

\subsection{La confiance au cœur de la mobilisation des utilisateurs et de leur collaboration}

Les Living Labs (LL) sont également un développement original, et relativement récent de l'IO [ARNKIL et al., 10], où les notions de confiance et de contrat sont des enjeux forts. En effet, les LL comme réseaux d'innovation supposent la mise en place de liens forts de coopération et de collaboration. Dans le cadre des LL, cette coopération se fait avec les utilisateurs qui sont placés au cœur des projets. D'expérience [DUPONT et al., 14, DUPONT, MOREL, GUIDAT, 15, DUPONT, MOREL, GUIDAT et al., 15, GUIDAT et al., 11], nous pouvons souligner que les projets en mode LL induisent notamment des contrats tacites entre les usagers participants, les porteurs de projets et les éventuels animateurs de la méthode sans pouvoir garantir la propriété intellectuelle (et/ou industrielle) de ce qui pourrait être produit. De plus, la mobilisation des acteurs se fait généralement sur une confiance mutuelle qu'il est nécessaire de construire et d'alimenter.

En transposant aux projets en mode LL les travaux sur les réseaux d'innovation au sein des projets d'entreprise [SHAZI et al., 15], nous admettons que les utilisateurs apportent des compétences différentes selon le sujet et le moment de la mobilisation (diagnostic, génération d'idées, retour d'expérience, etc.) pour apporter une expertise et des savoir-faire utiles au service de l'innovation. En revanche, dans tous les cas, leur bienveillance est requise et nécessaire. Elle reste un point nodal de la relation se construisant. Les recherches montrent que l'intégrité est également une caractéristique fondamentale. Un collaborateur compétent qui manque d'intégrité sera activement évité dans le montage et développement de projets d'innovation. Notre expérience des projets LL nous a confronté à certaines questions récurrentes de la part des participants utilisateurs par rapport aux commanditaires de projets: Que sera-t-il fait de nos contributions ? Servons-nous l'intérêt général ou un intérêt particulier ? Notre expression sera-t-elle respectée ? Etc. Réciproquement, les porteurs de projet, qui adoptent une approche LL visant à associer des utilisateurs dans leur démarche, se questionnent parfois sur l'intégrité des acteurs qui vont les rejoindre : respecteront-ils une certaine confidentialité si elle est exigée ? Adhèrent-ils à certaines de nos valeurs ? Seront-ils « sincères » ou encore « authentiques »? Etc. En pratique, comme il est impossible de vérifier a priori la compatibilité intégrale entre les participants, les animateurs et porteurs de projets, il faut accepter que des acteurs se retirent ou soient écartés au cours du processus. 


\subsection{Co-création et confiance : parents pauvres des technologies supports au collaboratif ?}

Une étude réalisée en 2018 sur les environnements immersifs collaboratifs [DUPONT et al., 18] offre un éclairage intéressant sur la notion de confiance et de co-création. Les auteurs ont trouvé et analysé 23 articles scientifiques publiées depuis 2000 dans des revues internationales ainsi qu'une thèse.

\begin{tabular}{|c|c|c|c|c|c|c|c|c|c|}
\hline \multirow[b]{2}{*}{ Auteurs } & \multirow[b]{2}{*}{ Année } & \multicolumn{2}{|c|}{ Fabrication du sens } & \multicolumn{2}{|c|}{$\begin{array}{c}\text { Construction de la } \\
\text { confiance }\end{array}$} & \multicolumn{2}{|c|}{ Signification partagée } & \multicolumn{2}{|c|}{ Compréhension mutuelle } \\
\hline & & $\begin{array}{c}\text { Compréhens } \\
\text { ion du } \\
\text { contexte }\end{array}$ & $\begin{array}{l}\text { Pertinen } \\
\text { ce }\end{array}$ & $\begin{array}{c}\text { Liens } \\
\text { Interpersonn } \\
\text { els }\end{array}$ & Confiance & $\begin{array}{c}\text { Partage de } \\
\text { Connaissance }\end{array}$ & $\begin{array}{l}\text { Création de } \\
\text { Connaissance }\end{array}$ & $\begin{array}{l}\text { Dynamique } \\
\text { de Groupe }\end{array}$ & $\begin{array}{l}\text { Intelligence } \\
\text { Collective }\end{array}$ \\
\hline [BENTE et al., 04] & 2004 & $\mathrm{Y}$ & $\mathrm{X}$ & $\mathrm{X}$ & $\mathrm{X}$ & & & $\mathrm{X}$ & $\mathrm{X}$ \\
\hline [NARAYAN et al., 05] & 2005 & $X$ & & & & & & $\mathrm{X}$ & $X$ \\
\hline [LOUCHART et al., 06] & 2006 & & & $X$ & & & & $X$ & \\
\hline [ANTONIAC et al., 06] & 2006 & $\mathrm{Y}$ & Y & $\mathrm{X}$ & & $\mathrm{X}$ & $\mathrm{X}$ & $\mathrm{Y}$ & Y \\
\hline [JENNETT et al., 08] & 2008 & $X$ & & & & & & & \\
\hline [PALLOT, 12] & 2012 & $X$ & $\mathrm{X}$ & $\mathrm{X}$ & $\mathrm{X}$ & $\mathrm{X}$ & $\mathrm{X}$ & $X$ & $X$ \\
\hline [LORENZO et al., 12] & 2012 & $\mathrm{Y}$ & $X$ & $\mathrm{X}$ & $X$ & $X$ & & $X$ & $X$ \\
\hline [DUPONT et al., 12] & 2012 & & & & & & & $X$ & $X$ \\
\hline [ŞTEFAN, 12] & 2012 & & & & & $\mathrm{X}$ & & & $\mathrm{Y}$ \\
\hline [SANTOS et al., 12] & 2012 & $X$ & $X$ & $X$ & & $\mathrm{X}$ & & $X$ & \\
\hline [CRUZ et al., 14] & 2014 & $X$ & & & & & & & \\
\hline [FREINA, OTT, 15] & 2015 & & & & & & & $X$ & \\
\hline [EYNARD et al., 15] & 2015 & & & & & & & $\mathrm{X}$ & $X$ \\
\hline [ORENSTEIN et al., 15] & 2015 & $\mathrm{Y}$ & $\mathrm{Y}$ & & & $\mathrm{Y}$ & & $\mathrm{Y}$ & $\mathrm{Y}$ \\
\hline$[$ Rотн, 15] & 2015 & & & $\mathrm{Y}$ & & & & & \\
\hline [Ke, CARAfano, 16] & 2016 & & & & & & & $\mathrm{X}$ & $\mathrm{X}$ \\
\hline [PALLOT, RichiR, 16] & 2016 & $\mathrm{Y}$ & & & & $\mathrm{Y}$ & $\mathrm{Y}$ & & $\mathrm{Y}$ \\
\hline $\begin{array}{l}\text { [POULIQUEN-LARDY, } \\
16]\end{array}$ & 2016 & $\mathrm{X}$ & $\mathrm{X}$ & & & $\mathrm{X}$ & $\mathrm{Y}$ & $\mathrm{X}$ & \\
\hline [ULEWICZ et al., 16] & 2016 & $\mathrm{X}$ & $\mathrm{X}$ & & & $\mathrm{Y}$ & & $\mathrm{Y}$ & \\
\hline $\begin{array}{l}\text { [GABANA ARELLANO } e t \\
\text { al. }, 16]\end{array}$ & 2016 & & & & & & & $X$ & $X$ \\
\hline [LE et al., 17] & 2017 & & & $\mathrm{Y}$ & & $\mathrm{Y}$ & $\mathrm{Y}$ & $\mathrm{Y}$ & \\
\hline [Fu, HwANG, 18] & 2018 & Y & & $X$ & & $X$ & & $X$ & $\mathrm{X}$ \\
\hline$[$ Du et al., 18$]$ & 2018 & & $\mathrm{Y}$ & $\mathrm{Y}$ & & & & $X$ & \\
\hline [LI et al., 18] & 2018 & & & & & $\mathrm{X}$ & & $\mathrm{X}$ & \\
\hline
\end{tabular}

$\mathrm{X}$ signifie une référence explicite à la propriété ; Y signifie une référence implicite à la propriété

Tableau 1. Analyse de la dimension eXperience Collaborative dans les publications traitant de plates-formes technologiques immersives depuis 2000 d'après [DUPONT et al., 18]

L'analyse (tableau 1) de ces études empiriques réalisées sur ces différentes plates-formes technologiques immersives et impliquant une diversité de participants, a permis de faire ressortir pour l'eXperience Collaborative (CX) quatre dimensions récurrentes : (1) la fabrication du sens ; (2) la construction de la confiance ; (3) la signification partagée ; (4) la compréhension mutuelle. Nous avons également identifié deux propriétés par dimension, respectivement : la compréhension du contexte et la pertinence; les liens interpersonnels et la confiance; le partage de connaissance et la création de connaissance ; la dynamique de groupe et l'intelligence collective.

La figure 1, ci-après, présente le nombre d'article qui font référence aux différentes propriétés de l'eXeperience Collaborative et révèle que deux propriétés sont peu éclairées par les études : la confiance et la création de connaissance. Le faible nombre d'études, relatives aux technologies de l'immersif collaboratif et s'intéressant à ces propriétés, questionne quant à l'importance accordée à des notions qui sont pourtant au cœur des processus collaboratifs, par essence ouverts et par nature inscrite dans un processus de création de connaissance, consubstantiel du processus d'innovation. 


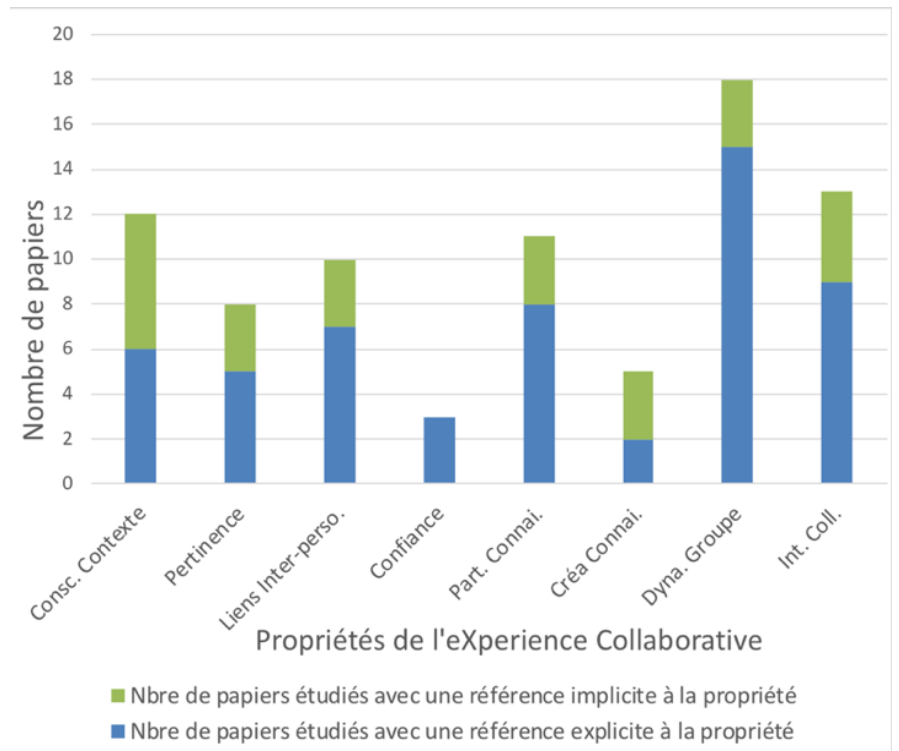

Figure 1. Nombre d'article du panel étudié citant une propriété ou un concept proche de l'eXeperience Collaborative d'après [DUPONT et al., 18]

Les différents éléments issus de la littérature soulignent le caractère fondamental de la confiance pour chacun des membres d'une entreprise à travers par exemple la croyance accordée en ses propres capacités. La confiance entre les membres d'une entreprise est également nécessaire pour garantir des relations équilibrées et profitables. La confiance doit ainsi se retrouver entre les niveaux hiérarchiques et fonctionnels. Enfin, la loyauté est un facteur incontournable dans les relations d'une organisation avec ses partenaires et les autres acteurs qui composent son environnement, notamment lorsqu'il s'agit d'associer les usagers dans la démarche. Plus globalement, il s'agit également d'avoir confiance dans son marché et sa capacité à s'adapter aux évolutions plus ou moins prévisibles.

Malgré la pertinence et la clarté de ces constats, il semble néanmoins toujours délicat d'établir et maintenir des relations de confiance. Bien que le contexte et la technologie peuvent répondre ponctuellement à un certain nombre de défis, il reste toujours des freins à dépasser pour renforcer la confiance aux différents niveaux que nous avons pu identifier. Dans une époque caractérisée notamment par la défiance envers le progrès scientifique et la technologie (l'affaire dite de la «vache folle », Tchernobyl ou la catastrophe de Fukushima, etc.), la remise en cause du journalisme («fakes news », etc.), la possible fragilité des réseaux sociaux (le scandale Cambridge Analytics), il semble nécessaire d'appréhender et d'outiller les processus de la phase amont de l'innovation pour garantir une innovation agile et ouverte pleinement consciente de ces défis sociétaux.

\section{Méthode : comparaison de cas d'étude issus de recherche exploratoires longitudinales}

Dans cet article nous adoptons une approche par cas d'étude [YIN, 13]. Cette méthode nous permet d'éclairer la question de la confiance à travers l'analyse complémentaire de deux cas d'études d'innovation ouverte appliquée au domaine de la distribution d'énergie pour deux pays européens (La Suisse et La France) qui ont tous les deux fait l'objet d'une initiative Living Lab sur une période relativement similaire. Le caractère novateur du déploiement de démarches d'IO pour les entreprises de ce secteur industriel nous incite à capitaliser et analyse des expériences concrètes pour développer et caractériser des méthodologies transposables. Ces travaux nous permet également d'apporter des analyses complémentaires aux recherches de [GRECO et al., 17] qui déplorent le faible nombre d'étude sur l'IO pour des entreprises de ce secteur.

Ainsi, le premier cas présente une partie des résultats d'une recherche exploratoire longitudinale menée lors de la création de " l'Energy Living Lab » en Suisse, dont l'enjeu est la création d'un écosystème d'innovation pour favoriser la co-création de services énergétiques pour un des 700 
distributeurs d'énergie. Ce projet, développé dans le cadre des travaux de thèse de Joëlle Mastelic, a été présenté à la conférence de l'Association Française de Marketing 2015.

Le second cas présente le projet français Link by Makers (LbM) de l'Université de Lorraine (UL). Ce projet impulsé par des universitaires et des makers (i.e. de membres investis dans un FabLab) a reçu le soutien d'ENEDIS, le distributeur français de l'électricité, comme moyen d'expérimenter une certaine forme d'IO autour du compteur communicant installé par le distributeur qui gère $95 \%$ du réseau français. Ce projet a été présenté à IEEE / ICE Conférence 2017 [DUPONT et al., 17].

Deux équipes différentes de chercheurs ont conçu ces cas de façon indépendante. La première équipe, impliquée dans un LL suisse, mène des travaux de recherche en marketing. La seconde, engagée dans un LL français, poursuit des travaux en ingénierie de l'innovation. Les deux cas d'étude que nous allons détailler ci-après ont émergé dans des contextes différents, néanmoins dans les deux cas les chercheurs ont considéré que les parties prenantes concernés dans chaque situation se faisaient confiance. Ils sont également partis du principe que les usagers faisaient confiance au processus mis en place par les universitaires pour générer une véritable dynamique de co-création. Les premiers travaux présentés indépendamment valident la capacité des processus à générer de la co-création. L'analyse croisée des retours d'expérience des deux équipes quant à la mise en place et au pilotage de ces deux cas nous permet de revenir sur le postulat de la confiance accordée a priori au processus par les parties prenantes. Cette dernière n'est pas automatique. Des freins peuvent être évités. Des bonnes pratiques ont été identifiées pour établir un climat de confiance entre parties prenantes d'un processus d'innovation ouverte sur des périodes de six à dix-huit mois. Enfin des leviers technologiques semblent pouvoir être utiliser pour accélérer ce processus d'innovation et lui garantir un caractère agile.

\subsection{L'Energy Living Lab : un écosystème d'innovation pour favoriser la co-création de services énergétiques}

Cette étude de cas, décrit un des premiers projets du Energy Living Lab (ELL). Il a débuté en automne 2013 et a duré 2 ans, dans un contexte de transition du secteur énergétique suisse. En effet, à cette période les distributeurs sont passés d'un statut monopolistique sur leur territoire de compétence, avec des clients captifs, à un contexte concurrentiel, dans le quels les clients sont libres de choisir leur distributeur d'énergie. L'enjeu de ce projet ELL est de mettre la co-création au service du développement de la relation clients et de la création de valeur pour l'entreprise partenaire. La méthode suivie est structurée en quatre étapes : la formulation du challenge, la plateforme de co-création, les publics cibles, la nature des données recueillies, ainsi que les étapes successives de traitement et d'analyse des données.

\subsubsection{La formulation du challenge}

La formulation du challenge, ou concours d'idées, a une grande importance. Elle doit être assez précise pour que le distributeur puisse développer les idées qui en résultent, mais également assez large pour permettre la participation des personnes sollicitées, qui ne proviennent principalement pas du domaine de la technique. La question a été définie par l'entreprise puis pré-testée auprès de 30 personnes. La question posée était la suivante : «L'efficience énergétique, concrètement, ça serait quoi pour vous ? De quels conseils, produits, services aimeriez-vous disposer pour réduire votre consommation d'énergie? Quelles prestations souhaiteriez-vous pour vous aider à la diminuer? ».

\subsubsection{La plateforme de co-création}

La plateforme de co-création www.i-brain.ch développée par l'entreprise suisse Atizo en partenariat avec la Haute École Spécialisée de Suisse Occidentale a été utilisée comme interface virtuelle. Cette plateforme permet (1) la génération d'idées en sollicitant un ou des groupes cibles, (2) la pré-sélection d'idées par un jury d'experts et (3) l'évaluation des idées présélectionnées par un ou des groupes cibles. Dans la phase de génération d'idées, l'objectif est de récolter un maximum d'idées auprès des 
populations cibles. Puis un jury d'experts sélectionne un nombre déterminé d'idées. Celles-ci sont ensuite soumises aux populations cibles qui les évaluent et les ordonnancent.

Les idées proposées sur la plateforme sont ouvertes et visibles, dans une optique de fertilisation croisée : les idées des uns viennent enrichir les idées des autres. L'intérêt de la plateforme réside également dans son automatisation: des rapports automatiques peuvent être tirés pour les phases (1) génération d'idées (2) sélection des idées et (3) évaluation des idées ce qui permet de gérer une grande quantité de données très rapidement.

\subsubsection{Publics cibles de la co-création}

Dans une optique de générer un maximum d'idées variées et de permettre la fertilisation croisée des idées, comme dans un brainstorming traditionnel, plusieurs publics-cible ont été sélectionnés. On recherche des usagers susceptibles d'être motivés à fournir des idées de nouveaux services énergétiques et également des usagers avec des idées innovantes. L'objectif n'est pas dans cette phase de génération d'idées d'être représentatif d'un certain échantillon de clients. D'autant, qu'au moment du challenge un client de l'entreprise de distribution en question ne se différencie d'un client d'un autre distributeur que par son lieu de résidence. Environ 500 personnes ont participé, issues des communautés suivantes :

- Les clients de l'entreprise : 30000 clients (ménages) ont été invités par email à donner des idées sur une plateforme en ligne. Cela représente $10 \%$ du total de ce type de clients. La plupart n'ont que peu de contacts avec l'entreprise (facture annuelle d'électricité) et peu de connaissance en matière d'énergie.

- Les membres de la communauté dédiée à l'efficacité énergétique : l'entreprise a développé un site internet qui propose de nombreuses informations en matière d'efficacité énergétique. 5000 personnes se sont inscrites de manière volontaire dans cette communauté et peuvent également partager leurs expériences. Ce ne sont pas forcément des clients de l'entreprise. On peut faire l'hypothèse qu'ils sont motivés à donner des idées en matière de services énergétiques et que ce sont des idées qui pourraient avoir de la valeur pour l'entreprise puisqu'ils sont informés sur le thème de l'efficacité énergétique. Ils ont également été invités par email à donner des idées.

- Les étudiants en Bachelor et Master de la HES-SO : 4707 étudiants en Bachelor et Master de la HES-SO ont été invités par email à participer sur une plateforme qui leur est dédiée. L'hypothèse faite par l'équipe de recherche est que les étudiants ont moins de filtres et vont peut-être proposer des idées moins normées que les clients et les membres de la communauté dédiée. Certes, la plupart d'entre eux ne payent pas encore de facture d'électricité mais ils consomment de l'énergie. La problématique de l'entreprise est plus centrée sur les besoins en matière de services qui pourraient aider les usagers à consommer moins que sur la commercialisation de ces services. La commercialisation devra bien entendu être définie dans une phase ultérieure d'élaboration de concepts puis de design de services et testée auprès de la cible.

Pour éviter de récolter des idées uniquement avec des clients ou des usagers connectés à internet, des interfaces physiques ont également été organisées pour collecter des idées lors de trois manifestations grand public pour lesquelles des hôtesses ont collecté des idées auprès des visiteurs par le biais de flyers.

\subsubsection{Traitement et analyse des données}

Le challenge a été proposé pendant un mois, de mi-septembre à mi-octobre 2014. Dans la phase de sélection des idées et afin de sélectionner les 30 meilleures idées à partir des idées initialement émises, deux jurys ont été constitués. Un jury d'experts internes à l'entreprise et un jury externe à l'entreprise. L'objectif de former deux jurys distincts et de les laisser totalement libres de choisir le processus de 
sélection des idées est de comparer les idées sélectionnées par les experts internes et celles des experts externes pour tester s'il y a une convergence ou une divergence, exprimée par le nombre d'idées communes aux deux jurys.

Le jury d'experts internes à l'entreprise (une experte de l'efficience énergétique, une experte en matière de communication liée à l'énergie et un responsable de ligne d'affaires) avait pour objectif de sélectionner les 15 meilleures idées selon le processus de leur choix. Celui-ci s'est décomposé en trois phases distinctes : une évaluation individuelle de chaque idée selon trois critères (pertinence de l'idée, caractère innovant de l'idée, faisabilité technique de l'idée), une mise en commun (pré-sélection) des idées retenues similaires et un tri final afin de définir les meilleures idées.

D'un autre côté, un jury externe et composé de professeurs de la Haute École Spécialisée de Suisse Occidentale travaillant dans le domaine de l'énergie a également eu la tâche de sélectionner les 15 meilleures idées. Les 12 critères pondérés de sélection définis par ce groupe sont présentés dans le tableau 2.

\begin{tabular}{lc} 
Critère & Poids \\
\hline Réalisable (technique / temps) & 5 \\
Compatible avec la législation & 3 \\
Impact social & 2 \\
Acceptabilité social & 4 \\
Impact économique / financier (collectivité) & 3 \\
Impact économique / financier (distributeur) & 5 \\
Impact économique / financier (consommateur) & 3 \\
Mobilité douce & 3 \\
Impact sur la production d'énergies renouvelables & 5 \\
Réduction de la consommation d'énergie (électricité) & 5 \\
Réduction de la consommation d'énergie (chaleur) & 5 \\
Temps de retour énergétique (coût/bn, efficience) & 4 \\
\hline
\end{tabular}

Tableau 2. 12 critères de sélection des idées

Par la suite, les 30 idées (15 provenant des experts internes et 15 des experts externes) sont soumises parallèlement aux deux groupes afin de comparer l'évaluation et l'ordonnancement des idées provenant des différents groupes.

\subsubsection{Résultats de l'étude empirique}

Les résultats des trois phases, (1) la génération d'idées (2) la sélection des idées (3) l'évaluation des idées, sont présentés et commentés ci-dessous.

\subsubsection{Résultats de la phase de génération d'idées}

L'objectif du distributeur d'électricité régional était de collecter au moins 300 idées soit $1 \%$ du nombre total de clients ayant été invités à participer par email. Ce sont les résultats empiriques moyens que l'on constate pour ce type de processus de co-création, dans les différents projets de recherche appliquée que nous avons menés. Il est donc important de pouvoir inviter un nombre conséquent de personnes à participer afin d'augmenter le nombre d'idées postées sur une plateforme de co-création.

Dans ce projet, 530 idées ont été collectées par les trois points de contact suivants: (1) 21 idées par les étudiants sur une plateforme internet dédiée (2) 436 idées par les usagers lors des trois manifestations via des flyers (3) 73 idées par les clients et les membres de la communauté dédiée à l'efficacité énergétique sur une autre plateforme internet dédiée. Une plus grande quantité d'idées a été collectée grâce aux interfaces physiques, lors des manifestations (436 idées) par rapport aux interfaces uniquement virtuelles (94 idées). 


\subsubsection{Résultats de la phase de sélection des idées}

Il ressort que sur les 15 idées sélectionnées par le jury d'experts de la HES-SO, 5 idées sont issues du groupe des étudiants et 10 idées du groupe des clients et autres usagers. Sur les 15 idées sélectionnées par le jury interne à l'entreprise, 4 idées sont issues du groupe des étudiants et 11 idées du groupe des clients et autres usagers.

Les processus d'évaluation radicalement différents, l'inégalité des critères choisis et la composition très dissemblable des deux groupes d'experts permettent d'expliquer en partie la divergence des avis exprimés sur les idées retenues et soulignent la démarche exploratoire de la présente étude. Par ailleurs, aucune des idées retenues n'est commune aux deux jurys.

\subsubsection{Résultats de la phase d'évaluation des idées}

Une fois les 30 idées sélectionnées, le processus d'évaluation des idées a pu débuter. Dans cette phase, qui a duré deux semaines, il était important de déterminer quelles pourraient être les idées qui pourraient être converties en produits, services et prestations qui pourraient être proposées par l'entreprise. Trois questions ont été définies comme critères d'évaluation des idées, en collaboration avec l'entreprise : «Comment jugez-vous cette idée ?», «Souhaiteriez-vous que ce service/produit soit mis en place pour votre ménage ? » et «Seriez-vous prêt à participer financièrement pour sa mise en place ? ». Ces trois questions permettent de comprendre si les idées proposées dans la phase initiale ont un avenir commercial pour l'entreprise et peuvent servir de base au développement de concepts produits/services dans une phase ultérieure.

Concrètement, 83 personnes issus de la population cible ont évalué les 30 idées retenues. Comme présenté dans le tableau 3, il ressort de cette évaluation que le total de points obtenus par les idées proposées par le groupe des étudiants oscille entre $6.72(\mathrm{~min})$ et $9.18(\mathrm{max})$, alors que celui du groupe des clients et autres usagers varie entre $5.45(\mathrm{~min})$ et $9.14(\max )$. A noter que le maximum de points était de 14. Les gagnants issus des 2 groupes ont été sélectionnés, informés personnellement et récompensés.

\begin{tabular}{|c|c|c|c|c|c|c|c|c|c|c|c|c|c|c|c|c|c|c|c|c|c|c|c|c|c|c|c|c|c|c|}
\hline \multirow{2}{*}{$\begin{array}{l}30 \text { idées sélectionnées } \\
\text { Comment jugez-vous cette idée } \\
\text { (Moyenne) }\end{array}$} & \multicolumn{21}{|c|}{ 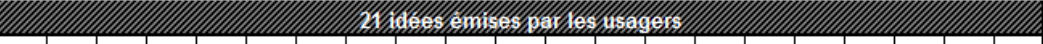 } & \multicolumn{9}{|c|}{9 idées émises par les étudiants } \\
\hline & 3.2 & 3.5 & 3.4 & 3.8 & 3.3 & 3.3 & 2.8 & 3.7 & 3.1 & 4.2 & 2.4 & 3.8 & 3.5 & 3.5 & 3.5 & 3.5 & 4.2 & 3.9 & 4.1 & 2.7 & 3.5 & 3.4 & 3.7 & 3.6 & 3.1 & 4.1 & 3.1 & 3.5 & 4.4 & 4.5 \\
\hline $\begin{array}{l}\text { Souhaiteriez-vous que ce } \\
\text { service/produit soit mis en place pour } \\
\text { votre ménage? } \\
\text { (Moyenne) }\end{array}$ & 2.1 & 2.4 & 2.4 & 2.6 & 2.3 & 2.2 & 1.9 & 2.5 & 2.1 & 2.9 & 1.7 & 2.6 & 2.4 & 2.4 & 2.4 & 2.4 & 2.9 & 2.6 & 2.7 & 2.0 & 2.4 & 2.4 & 2.6 & 2.4 & 2.1 & 2.9 & 2.1 & 2.4 & 2.9 & 3.1 \\
\hline $\begin{array}{l}\text { Seriez-vous prêt à participer } \\
\text { financièrement pour sa mise en place? } \\
\text { (Moyenne) }\end{array}$ & 1.6 & 1.6 & 1.7 & 2.1 & 1.8 & 1.6 & 1.4 & 1.8 & 1.6 & 1.8 & 1.4 & 1.9 & 1.9 & 1.7 & 1.8 & 1.8 & 2.1 & 2.0 & 1.9 & 1.6 & 1.7 & 1.9 & 1.9 & 1.8 & 1.6 & 2.2 & 1.6 & 1.8 & 1.9 & 2.3 \\
\hline Somme des points & 6.91 & 7.58 & 7.43 & 8.56 & 7.48 & 7.04 & 6.10 & 8.01 & 6.75 & 8.85 & 5.45 & 8.31 & 7.79 & 7.54 & 7.66 & 7.76 & 9.14 & 8.44 & 8.69 & 6.23 & 7.63 & 7.72 & 8.20 & 7.81 & 6.85 & 9.14 & 6.72 & 7.64 & 9.10 & 9.88 \\
\hline Classement & 17 & 12 & 15 & 4 & 14 & 16 & 20 & 7 & 18 & 2 & 21 & 6 & 8 & 13 & 10 & 9 & 1 & 5 & 3 & 19 & 11 & 6 & 4 & 5 & 8 & 2 & 9 & 7 & 3 & 1 \\
\hline
\end{tabular}

Tableau 3. Notes moyennes des évaluations des 30 idées sélectionnées, sur les trois critères d'évaluation

Sur les 30 idées sélectionnées par les deux jurys d'experts, 9 proviennent du processus de génération effectué auprès des étudiants, dont l'idée ayant obtenu la meilleure note et celle ayant obtenu la $2^{\mathrm{e}}$ meilleure note (à égalité). Dans le cas de cette entreprise romande, le processus réalisé auprès d'un public d'étudiants qui ne constitue pas sa clientèle à court terme a été une richesse ; sur 21 idées émises par les étudiants 9 idées (soit 43\%) ont été sélectionnées par les jurys. Sur les 509 idées émises par les clients et usagers, 21 (soit 4\%) ont été sélectionnées pour l'évaluation finale. Avoir réalisé ce travail en parallèle avec deux groupes distincts a donc été intéressant ; le groupe des étudiants, habitué et formé à être sollicité afin de générer des idées et des solutions, a été une source importante d'idées valorisées par les deux jurys. 
Il serait intéressant de pouvoir mesurer cette différence dans l'appréciation des idées entre un groupe d'usagers et un groupe d'étudiants lors d'une étude approfondie. Le mélange des groupes s'est dès lors révélé être un choix méthodologique judicieux.

Par contre, en analysant plus en détails les moyennes des notes des 30 idées qui ont été évaluées, on s'aperçoit grâce à un test de Student que la moyenne des notes des idées émises par les étudiants $(8.12$ points) n'est pas significativement différente de la moyenne des notes des idées émises par les clients/usagers (7.59). De même pour la moyenne des notes du jury interne (7.38) qui n'est pas significativement différente de la moyenne des notes du jury externe (8.11), la moyenne globale étant de 7.74 points. Ce qui laisserait penser que les jurys ont sélectionné plus d'idées provenant des étudiants en relation avec le nombre d'idées soumises mais que dans cette étape d'évaluation des 30 idées sélectionnées, les idées provenant des deux publics-cibles sont en moyenne de qualité égale.

Pour des raisons de confidentialité, l'entreprise n'a pas souhaité partager la liste complète des idées avec leurs évaluations respectives mais a communiqué sur les trois idées gagnantes, dans le cadre d'un communiqué de presse.

\subsubsection{Mise en perspective des résultats ELL en termes de confiance}

L'intérêt des usagers pour la co-création a été mesuré par le nombre d'idées soumises par les usagers dans les différents contextes (interfaces physiques et virtuelles). Le nombre de réponses souhaitées par le partenaire économique a été atteint, mais il a été nécessaire de passer de l'interface virtuelle à une interface physique afin de stimuler les usagers en face à face (voir tableau 4). Le challenge présenté a dû être expliqué lors de ces interactions, ce qui pourrait laisser entendre que la question était trop technique ou complexe, et qu'elle n'a pas était appropriée par les usagers ce qui a pu représenter un frein pour la participation via l'interface virtuelle. L'équipe de recherche espérait que le taux de participation grâce aux interfaces virtuelles soit plus élevé. Leur confiance dans la capacité des usagers à se saisir individuellement et virtuellement de cette question était surévaluée.

\begin{tabular}{|c|c|c|c|c|}
\hline & $\begin{array}{l}\text { Clients du } \\
\text { distributeur }\end{array}$ & $\begin{array}{l}\text { Membres de la } \\
\text { communauté dédiée à } \\
\text { l'efficacité énergétique }\end{array}$ & $\begin{array}{l}\text { Étudiants en Bachelor et } \\
\text { Master de la HES-SO }\end{array}$ & Total \\
\hline $\begin{array}{l}\text { Nombre dans la } \\
\text { population }\end{array}$ & 30000 & $\begin{array}{l}5000 \text { (dont certains } \\
\text { peuvent être issus des } \\
\text { clients) }\end{array}$ & 4707 & Entre 30000 et 39707 \\
\hline Participants & \multicolumn{3}{|c|}{ Environ 500} & Environ 500 \\
\hline $\begin{array}{l}\text { Idées déposées via } \\
\text { des plates-formes } \\
\text { internet }\end{array}$ & \multicolumn{2}{|c|}{73} & 21 & \multirow[t]{2}{*}{530} \\
\hline $\begin{array}{l}3 \text { Manifestions } \\
\text { dédiées }\end{array}$ & \multicolumn{2}{|c|}{436} & Non-Concernés & \\
\hline $\begin{array}{l}\text { Origine des idées } \\
\text { sélectionnées }\end{array}$ & \multicolumn{2}{|c|}{21} & 9 & 30 \\
\hline $\begin{array}{l}\text { Moyenne des notes } \\
\text { attribuées (via test } \\
\text { de Student) }\end{array}$ & \multicolumn{2}{|c|}{7.59 points } & 8.12 points & 7.74 points \\
\hline
\end{tabular}

Tableau 4. Synthèse des résultats du processus de co-création mis en œuvre par ELL

Les idées sélectionnées par l'entreprise soulèvent néanmoins plusieurs questions techniques, juridiques ou opérationnelles et l'entreprise réalise des analyses complémentaires afin d'en confirmer la faisabilité et, le cas échéant, les modalités de mise en œuvre. Il convient néanmoins de souligner qu'aucune idée proposée n'a été considérée comme étant réellement nouvelle et nombreuses d'entre elles étaient redondantes, ce qui n'est pas surprenant dans une phase de génération d'idées où l'on cherche à comprendre les besoins latents des usagers. Ces idées brutes doivent ensuite poursuivre un long chemin dans le processus d'innovation pour être transformées en concepts puis en prototype et 
enfin en services. Ce qui questionne quant au réel apport de valeur par les différentes parties. La phase critique est de continuer le design du service après cette phase d'idéation à l'interne de l'entreprise, c'est à ce moment que peut apparaître le risque du «not invented here syndrome » [CHESBROUGH, 11] qui se caractérise par le rejet des idées non générées au sein de l'entreprise et bloquant le processus.

La démarche entreprise souligne la volonté clairement affichée par l'entreprise d'intégrer l'utilisateur au centre des réflexions; les profondes mutations que subit le marché de l'énergie en Suisse impactent directement la culture interne de l'entreprise et une véritable stratégie d'ouverture globale permet de la préparer à la libéralisation du marché (la mise en place d'une plateforme internet d'échange, à la fois informative, incitative et collaborative témoigne aujourd'hui de cette démarche globale initiée).

Les interfaces physiques semblent apporter un plus grand nombre d'idées que les interfaces uniquement virtuelles. Trois hypothèses pourraient expliquer ce point : (1) l'énergie n'est pas «top of mind» auprès des usagers interrogés et il faut stimuler le groupe en face à face pour qu'elle donne des idées, (2) le challenge posé était mal formulé et peu compréhensible pour des non experts, (3) l'interface virtuelle n'offre pas suffisamment de garanties par rapport à l'incertitude générée par la démarche (nécessité de rassurer et répondre rapidement aux interrogations ou incompréhensions que facilitent les rapports physiques directs).

Le groupe sollicité semble avoir une influence sur le nombre et la qualité des idées soumises. Le groupe des étudiants a soumis plus d'idées au prorata des personnes invitées et les idées provenant de ce groupe ont été jugées de meilleure qualité (plus d'idées sélectionnées au prorata des idées soumises). L'entreprise analysée a débuté depuis peu la mutation de sa relation avec ses clients en vue de la prochaine libéralisation du marché de l'électricité pour les ménages en Suisse. Il est possible que la relation de confiance et de fidélisation ne soit pas encore suffisamment forte pour que les clients s'engagent dans une co-création via une plateforme virtuelle. Car comme le souligne [CovA, CovA, 09] «le processus de gouvernementalité sous-jacent au discours du nouveau consommateur doit rappeler aux entreprises qu'il ne faut pas qu'elles croient que tous leurs clients ont succombé au formatage du consommateur créatif et qu'ils possèdent tous aujourd'hui des compétences pour dialoguer, jouer un rôle et intégrer les offres de l'entreprise », ce processus prend du temps et nécessite plusieurs itérations avant de pouvoir transformer ses clients en agents de changement.

Les avis divergents des groupes d'experts peuvent s'expliquer par diverses hypothèses ; le jury interne à l'entreprise est sous l'influence d'une stratégie interne latente, d'un réalisme quant à l'applicabilité des idées suggérées ou d'une réflexion «time-to-market». Rien ne permet toutefois d'affirmer que la composition de deux jurys externes et homogènes aurait mené à des sélections d'idées convergentes : la flexibilité laissée aux jurys quant à l'organisation du processus de sélection accentue la démarche exploratoire d'une telle étude. Cela peut également être issu du syndrome «not invented here » cité plus tôt. Pourtant l'intérêt d'aller chercher des idées externes à l'entreprise, comme le rappelle Chesbrough, est de dépasser ce syndrome en travaillant sur l'ouverture, notamment dans un environnement monopolistique. Nous retrouvons dès lors la question de la confiance des membres de l'entreprise vis à vis de l'extérieur.

Si le cas d'étude Suisse permet d'appréhender finement la dimension immatérielle de la créativité à travers un processus de génération d'idée brute, le cas d'étude français sur le compteur communicant nous permet d'approfondir le passage de l'idée à son prototypage dans un contexte de mobilisation d'acteurs très éclectiques parmi une population non clairement identifiée.

\subsection{Cas d'étude 2 : Linky by makers, un projet d'Ol entre industriel, universitaire, makers}

\subsubsection{Contexte du projet et public cible}


Linky by Makers (LbM) questionne les usages des compteurs communicants et des réseaux intelligents. Ce projet a débuté fin 2015 lors d'un échange entre industriels, universitaires et makers au sein du Lorraine Fab Living Lab ${ }^{\circledR}$ (http://www.lf21.fr/), plate-forme d'évaluation prospective des usages et d'acceptabilité des innovations du laboratoire ERPI et de l'ENSGSI, École Nationale Supérieure du Génie des Systèmes et de l'Innovation [DUPONT, MOREL, LHOSTE, 15]. LbM a reçu le soutien d'ENEDIS et de l'UL dans la continuité d'une collaboration amorcée dès 2009 et renforcée par la Chaire REVES (Renaissance Écologique des Villes) [DUPONT, MOREL, GuIDAT, 15]. Sur l'impulsion de makers nancéiens en réseau avec d'autres makers français, ce projet devait durer 6 mois mais a connu de nombreux "soubresauts" sur lesquels nous reviendrons car il est question de confiance.

Entre 2013 et 2022, ENEDIS doit déployer 35 millions de compteurs communicants sur le territoire français. Ces compteurs sont la propriété du distributeur d'électricité. Le compteur en lui-même est en phase d'installation en vue de son exploitation commerciale. Le produit «compteur communicant» peut être qualifié de «fini » mais le système de réseau électrique «intelligent» ne sera pleinement opérationnel que lorsque tous les compteurs seront installés sur le territoire français. Nous ne reviendrons pas directement ici sur la polémique soulevée par le déploiement de cette technologie [ASSEMBLEE NATIONALE, 16]. En revanche, la transition technologique et industrielle en cours et les questions qu'elle soulève font le contexte du projet.

LbM s'inscrit dans une logique d'innovation ouverte basée sur le principe qu'universitaires, consommateurs et fournisseurs peuvent travailler ensemble [CARAYANNIS, CAMPBELL, 12], notamment pour appréhender les usages d'une technologie encore peu connue pour le plus grand nombre bien que nous soyons tous utilisateur de l'électricité. Le projet expérimental pose l'hypothèse suivante : les makers qui ont l'habitude de «bidouiller» pourraient: (1) être une communauté avant-gardiste qui enrichit la compréhension des usages possibles du compteur (via des développements supplémentaires en open hardware) ; (2) Soutenir une réflexion sur les nouvelles formes de production / consommation / usage de l'électricité des réseaux dits intelligents.

Le projet devait alors permettre aux FabLabs français, ou ceux qui se considéraient comme makers, et qui étaient intéressés par la question de l'énergie, de s'autosaisir de ce défi. L'UL, via le laboratoire ERPI, se positionne en support de recherche pour aider les acteurs à communiquer entre eux, partager les idées, capitaliser et analyser l'expérience.

Lancé dans 5 régions française via 5 FabLabs "Régionaux" (FLR), le projet devait s'achever en juin 2016. Or, il y a eu une sous-estimation du temps nécessaire à la préparation des conditions de succès du projet. Ex : il y a eu plusieurs mois de décalage entre la signature des conventions entre les FLR partenaire et 1'UL pour leur attribuer des fonds (frais de déplacement des makers, achat de matériel, etc.), il y a eu une imprécision sur les modalités de fonctionnement, les attendus, etc. ceci ayant contribué à une forte démotivation qui a profondément remis en cause la poursuite du projet.

Après un constat de crise en juin 2016, un nouveau départ a été impulsé en septembre 2016 sur la base d'une analyse des premiers mois, une compréhension des difficultés, une validation des partenaires toujours motivés... L'enjeu était de mieux considérer les pratiques et rythmes de chacun tout en instaurant une méthode soutenable d'ingénierie collaborative distribuée. Nous avons également ouvert le réseau des participants en proposant aux étudiants de l'association FabLab des étudiants de l'ENSGSI, le GSI Lab qui a accès au LF2L. Finalement, les partenaires de LbM se sont engagés jusqu'à fin juin 2017 en signant une convention commune (6 partenaires signataires). A l'issue de cette période, 30 fiches idées ont été produites, 8 prototypes ont été développés et expérimentés. Ces prototypes, plutôt liés à la vie quotidienne ont pour objectifs de faciliter l'usage des fonctionnalités de Linky et constituent la base de projets potentiels. Les codes associés à ces prototypes sont librement accessibles sur internet. En revanche, deux projets à plus forte ambition et relative à la mise en place de systèmes communautaires ont été suggérés par les makers. 
En parallèle, les universitaires ont présenté leur résultat scientifique lors d'une conférence et ils ont remis à ENEDIS un rapport technique exhaustif décrivant les résultats du processus de co-création ainsi que la méthode ad hoc développée de façon agile en matière d'ingénierie collaborative ouverte. L'ensemble des résultats du projet ont été présenté à des entrepreneurs et citoyens intéressés par les questions énergétiques lors du $2^{\text {ème }}$ Forum des "Transitions et Gouvernance(s) de l'Énergie" (Avril 2018, Lyon) en lien avec l'initiative Daisee, d'internet de l'énergie (https://daisee.org/). Ces différentes rencontres semblent conduire à une mise en réseau de projets et d'énergie individuelle.

\subsubsection{Technologies déployées pour soutenir la co-création}

En procédant de manière itérative et constructiviste, l'équipe de recherche, en interaction avec les participants, a déployé et accompagné ou adapté l'usage des technologies collaboratives suivantes :

- La plate-forme collaborative Slack (https://bymakers.slack.com) permet de capitaliser et croiser les réflexions, questions et suggestions de tous les participants (makers, universitaires, groupe industriel).

- La plate-forme de créativité, dépôt des fiches idées, «48h Innovation makers » (http://ensgsi.kalanda.info/ENSGSI) mise à disposition par l'ENSGSI, permet aux participants (makers, universitaires, industriels) de déposer et consulter toutes les idées et projets imaginés. Un système de note ( 1 à 5 étoiles) permet aux participants de voter pour les idées.

- Le LF2L est mis à la disposition du NYBI (FLR Est) et du FabLab associatif GSI Lab une demijournée par semaine, avec augmentation possible de la fréquence au lancement du projet. Le LF2L accueille les événements spécifiques organisés avec les makers (Lancement LbM, Hackathon «énergie », point d'étape avec ENEDIS) et il sert aux universitaires engagés dans la réalisation de prototypes (usage sans restriction, à la demande des volontaires). A titre d'exemple, 9 ateliers de créativité puis de prototypage ont été organisés par le laboratoire ERPI lors du $1^{\mathrm{er}}$ semestre 2017.

La plate-forme Github (https://github.com/LF2L) est utilisée pour capitaliser et présenter les projets de prototype. Ces éléments produits sous licence «open source hardware » sont partagés et peuvent faire l'objet d'amélioration par l'ensemble de la communauté intéressé par les compteurs communicants et les Smart Building. Cette plate-forme permet dès lors de renforcer la co-conception entre les participants (makers, universitaires, industriels).

\subsubsection{Mise en perspective des résultats $L b M$ en termes de confiance}

Finalement, le principal défi de LbM consiste à faire collaborer entre eux des acteurs répartis sur le territoire français, aux temporalités différentes (temps plein et/ou contribution ponctuelle, salariés ou bénévoles, etc.), aux pratiques différentes, etc. En d'autres termes, il s'agit d'articuler 4 dimensions [DUPONT et al., 17] : (1) le Géographique avec des FLR dans 4 régions différentes, eux-mêmes composés d'acteurs distribués dans leur territoire conduisant à un manque d'interactions face à face (manque de spontanéité) ; (2) le Conceptuel car «créativité », «énergie », « open source / hardware » ne renvoie pas aux mêmes représentations et usages selon les acteurs, de plus le projet a débuté par une approche en créativité (i.e. produire des concepts abstraits avec une méthode universitaire) alors que les makers sont dans le «faire» (démonter, remonter, reproduire, transformer, etc.), enfin LbM a rassemblé et confronté des organisations structurées et des organisations très agiles ou floues aux modes de gouvernances parfois contradictoires ; (3) le Technologique avec une limite initiale forte, le distributeur n'a pas souhaité confier des compteurs communicants et les participants n'avaient pas de connaissance technique poussée sur cette technologie ou sur d'autres aspects du projet (mesurer l'électricité, développer des objets en open hardware, travailler en open source, etc.) ; (4) le Temporel qui se décline à travers le temps disponible des participants selon s'ils peuvent s'investir sur 6 mois, 2 ans, le temps d'un week-end, etc. Le temps concerne aussi le management des connaissances produites : comment laisser des contributions que d'autres pourront reprendre et valoriser? 
Au-delà de ces quatre barrières à dépasser pour assurer du collaboratif, ce cas d'étude permet de souligner trois constats liés au processus d'identification et de co-création des usages de l'électricité distribuée par des réseaux communicants : (1) Chacun a ses pratiques et objectifs propres : une entreprise "d'ingénieurs" avec une stratégie industrielle, des universitaires avec un projet de recherche, des FabLabs avec leurs histoire et logique d'action spécifiques et des centres d'intérêts différents, etc. ; (2) Les makers (au moment du projet LbM) sont finalement peu intéressés ou habitués aux questions énergétiques, par ailleurs, il est nécessaire d'apprendre à donner une expression concrète à des éléments intangibles (énergie, électricité, confiance, donnée, communication, etc.) ; (3) L’Intégration des utilisateurs en phase de lancement de produit, lorsque les décisions stratégiques irréversibles ont été prises, est trop tardive ce qui inhibe l'auto-détermination et la motivation à s'inscrire dans un processus de co-création. Il s'agit de co-construire une vision partagée par tous les acteurs en phase amont.

A l'image du contexte suisse du ELL, le contexte français a largement conditionné les prédispositions des acteurs à collaborer, nécessitant de transcender des pratiques et des centres d'intérêts individuels et spécifiques fortement ancré dans chacun pour construire une communauté d'intérêt dans un contexte flou et tendu [ASSEMBLEE NATIONALE, 16].

Finalement, nous pouvons résumer le pilotage du projet LbM en 5 défis qu'il a fallu surmonter pour créer un climat propice de collaboration entre les acteurs : (1) Avoir une représentation commune du projet; (2) Garantir les interactions entre communautés au bon moment dans un processus en construction ; (3) Développer des compatibilités de gouvernance par négociation de pairs à pairs ; (4) Gérer la communication interne et externe ; (5) Donner une explication concrète à des éléments intangibles et recourir à différents médias pour matérialiser à la fois le processus projet et ses productions. Nous avons conscience du caractère non exhaustif de démarche à entreprendre, en particulier pour les questions de transition énergétique. Les travaux de [KOIRALA et al., 18] soulignent notamment l'importance des communautés locales pour impliquer les citoyens et usagers dans le développement de réponses nouvelles comme systèmes énergétiques communautaires. Cette étude montre d'ailleurs que la confiance accordée à la communauté locale est un des facteurs déterminant pour s'engager.

\section{Vers un système support à la confiance dans la co-création}

\subsection{Enseignements issus des deux études de cas}

Les deux expérimentations que nous avons menées et étudiées nous permettent de mettre en en exergue les défis relatifs à la mise en confiance des parties prenantes aux différents niveaux d'un projet d'Innovation Agile et Ouverte. Au-delà des limites rencontrées dans la mise en œuvre des projets, leur observation et analyse nous donnent l'opportunité de comprendre plus finement les mécanismes en œuvre entre des communautés distribuées et sollicitées pour contribuer à un projet commun. L'engagement des chercheurs pour la conception, l'installation et l'animation des processus et le caractère extensif des analyses que nous avons pu produire à partir de ces deux projets, respectivement de 24 et 18 mois, nous permettent d'accéder à une lecture tant quantitative que qualitative de ces matériaux de recherche originaux. La figure 2 repend ainsi les principaux éléments technologiques déployés pour soutenir la co-création lors des projets ELL et LbM et favoriser la confiance entre les acteurs. Les plates-formes physiques ou virtuelles mobilisées sont classées par ordre d'apparition dans le processus de chacun des projets. Ce schéma indique par ailleurs les productions quantitatives issues de chacune d'entre elles. 


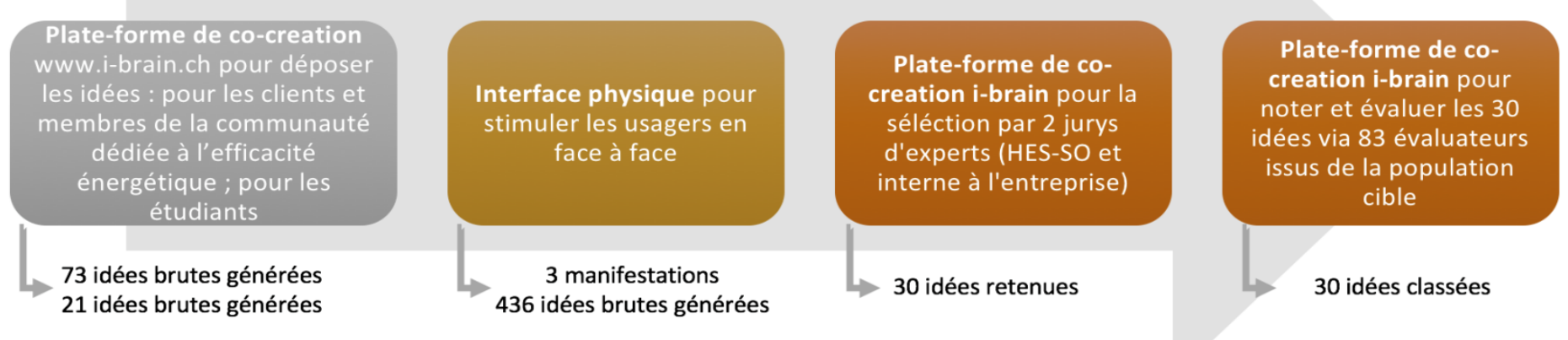

Cas d'étude 2 observé : LbM (18 mois)

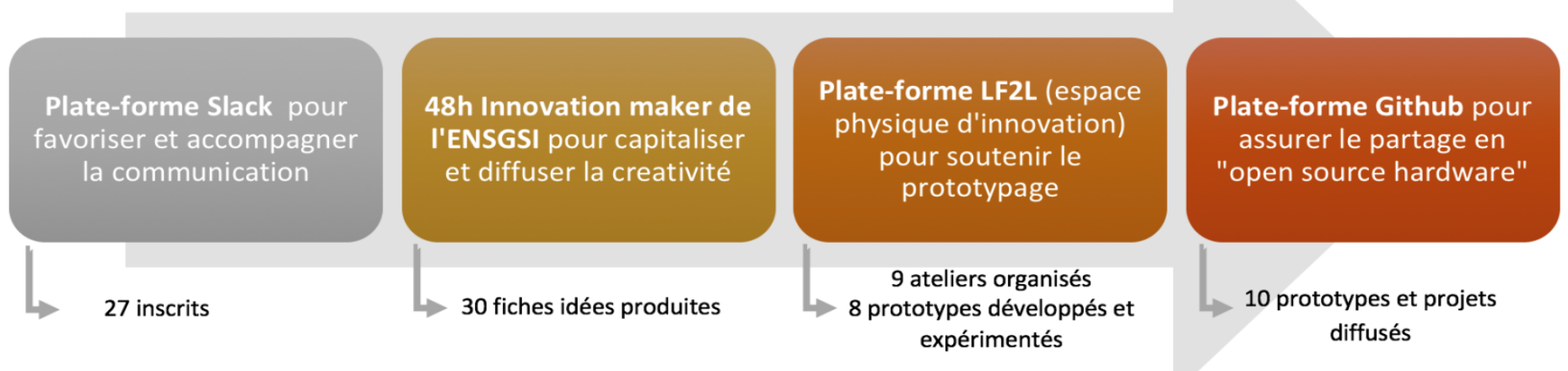

Figure 2. Vision séquentielle de la complémentarité des plates-formes support à la co-création au sein des projet ELL et LbM (d'après [DUPONT et al., 17])

Sur la base des observations et analyses rapportées au chapitre 3, nous pouvons améliorer chacun de ces processus afin de renforcer la co-création entre les acteurs. La figure 3 décrit ainsi les technologies ou fonctionnalités supplémentaires qu'il semblerait intéressant d'ajouter compte tenu du retour d'expérience. Il apparaît que deux fonctions ne sont encore remplies par aucun des projets : l'appropriation au sein des entreprises des idées générées à l'extérieur et la capacité à fidéliser et engager des consommateurs ou des communautés dans un processus de co-création impliquant une entreprise. Dans les deux cas, il faut d'ailleurs souligner que des universitaires ont sans doute joué un rôle de tiers de confiance vis-à-vis du processus et des problématiques soumises aux usagers. La proposition de soutien au design de service imaginé par ELL peut être assimilé à la pratique du LF2L et mis en œuvre pour le projet LbM. Enfin, la perspective de voir émerger une plate-forme connectant bâtiments, consommateurs et producteurs est spécifique au cas français, donc pour le moment peu généralisable.

Pour chaque plate-forme et chaque technologie, il est nécessaire de s'interroger sur la confiance que leur accorde les utilisateurs, ainsi que le rôle tenu par ces technologies sur le niveau de confiance que s'accordent les parties prenantes entre elles. En quoi l'intégration et la mise en œuvre de ces technologies génèrent-elles un climat favorable entre parties prenantes pour renforcer la confiance mutuelle et collaborer ensemble ? En d'autres termes, en reprenant les éléments de la littérature relatifs à la confiance, comment s'assurer que ces technologies encouragent un processus d'Innovation Ouvert : (1) la bienveillance ; (2) la capacité à remplir les «obligations » convenues entre parties ; (3) le partage et l'adhésion à des objectifs clairs et appréciés ; (4) l'émergence d'un environnement non menaçant où les parties peuvent contribuer et influencer les décisions en étant reconnues et valorisée dans leur contribution ; (5) le souci d'atteindre l'excellence grâce à un travail de qualité et à une 
évaluation critique ; (6) la valorisation de l'innovation et le soutien de pratiques de travail visant à réaliser l'innovation.

Simulation d'évolution des technologies à partir du cas ELL

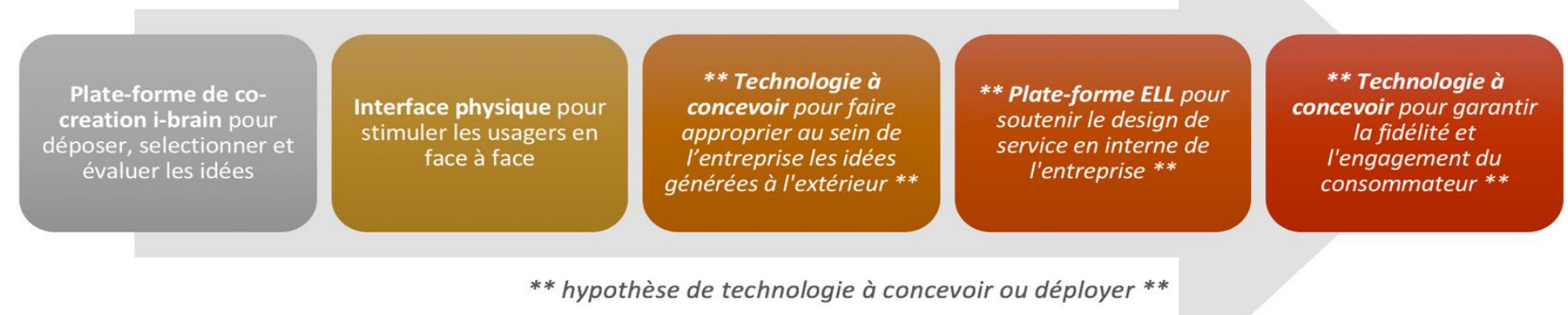

Simulation d'évolution des technologies à partir du cas LbM

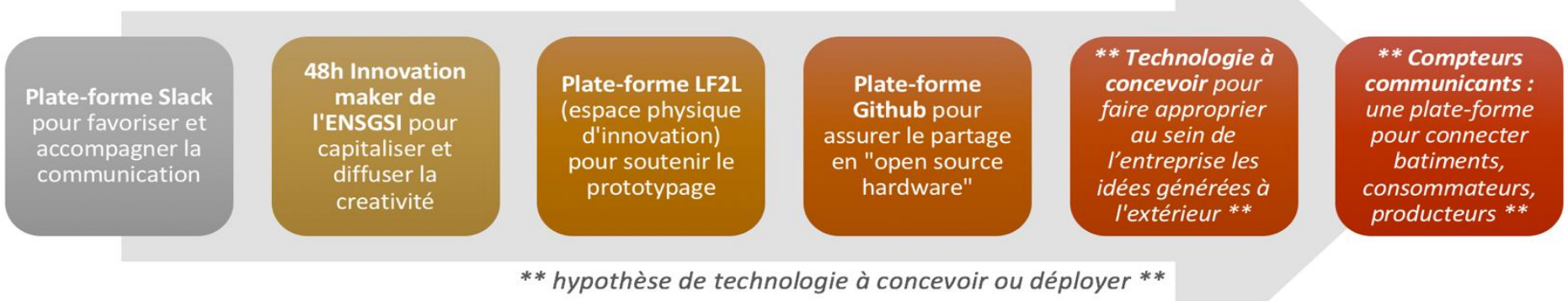

Figure 3. Proposition d'évolution des supports technologiques pour renforcer la co-création pour chacun des projets ELL et LbM

\subsection{Esquisse d’un processus intégré au service de la co-création}

Au regard des constats précédents, nous pouvons esquisser un processus doté de nouvelles réponses technologiques. La figure 4 ci-après propose ainsi une vision intégrée des bonnes pratiques permettant d'établir un climat de confiance entre les parties prenantes d'un processus d'innovation ouverte. Ce processus potentiel est construit par agrégation et ordonnancement des retours d'expérience issus des deux cas d'études. Nous avons identifié huit grandes fonctions que doit assurer une technologie ou un consortium de technologies collaboratives pour renforcer la co-création et la confiance dans un processus d'Innovation Agile et Ouvert: Communication, Idéation, Matérialisation, Contribution, Appropriation, Validation, Rétribution, Publication. Les définitions que nous retenons pour ces fonctions proviennent des cas étudiés et de notre expérience dans le domaine du pilotage de projet de co-création. La présentation des fonctions de manière séquentielle reprend l'ordre de survenu observé sur les cas pratiques, sans pour autant figer ces étapes qui peuvent parfois être menée concomitamment. Des boucles de rétroaction, ou a minima des itérations, doivent également être envisagée.

Pour caractériser et affiner les huit fonctions que doit assurer une technologie ou un consortium de technologies collaboratives, nous les avons croisées avec les déterminants de la confiance au sein d'un processus d'innovation. Cette méthode nous permet d'esquisser une matrice, nommée «co-con » que nous avons complété à partir des travaux présentés dans les chapitres précédents. Le contenu de la matrice constitue une première base de réflexion et de travail à destination des chercheurs et des praticiens comme guide de bonnes pratiques et pistes possibles de mise en œuvre. 
Vers une technologie encouragent un processus d'Innovation Ouvert et Agile?

\begin{tabular}{|c|c|c|c|c|c|c|c|c|}
\hline & $\begin{array}{l}\text { Favoriser et } \\
\text { accompagner la } \\
\text { communication } \\
\text { physique et } \\
\text { virtuelle }\end{array}$ & $\begin{array}{l}\text { Déposer, } \\
\text { selectionner et } \\
\text { évaluer les } \\
\text { idées }\end{array}$ & $\begin{array}{c}\text { Soutenir le } \\
\text { prototypage } \\
\text { collaboratif de } \\
\text { produits et de } \\
\text { services }\end{array}$ & $\begin{array}{l}\text { Assurer le } \\
\text { partage en } \\
\text { "open source } \\
\text { hardware" des } \\
\text { co-créations }\end{array}$ & $\begin{array}{c}\text { Faire } \\
\text { s'approprier } \\
\text { au sein des } \\
\text { entreprises les } \\
\text { idées générées } \\
\text { à l'extérieur }\end{array}$ & $\begin{array}{l}\text { Capitaliser les } \\
\text { tests par } \\
\text { l'usage (et le } \\
\text { retour } \\
\text { d'expérience) }\end{array}$ & $\begin{array}{l}\text { Fidéliser et } \\
\text { engager les } \\
\text { communautés }\end{array}$ & $\begin{array}{l}\text { Garantir la } \\
\text { transparence }\end{array}$ \\
\hline Confiance $\quad$ Fonctions & Communication & Idéation & Matérialisation & Contribution & Appropriation & Validation & Rétribution & Publication \\
\hline Bienveillance & $\begin{array}{l}\text { Au sein des } \\
\text { communautés } \\
\text { et entre les } \\
\text { communautés }\end{array}$ & $\begin{array}{l}\text { Laisser } \\
\text { s'exprimer les } \\
\text { idées }\end{array}$ & $\begin{array}{l}\text { Confier la mise } \\
\text { en œuvre à un } \\
\text { tiers }\end{array}$ & $\begin{array}{l}\text { Laisser les } \\
\text { autres modifier } \\
\text { son idée }\end{array}$ & $\begin{array}{l}\text { Valorisation des } \\
\text { idées, méthodes } \\
\text { agiles }\end{array}$ & $\begin{array}{l}\text { Droit à l'erreur } \\
\text { pour soi et pour } \\
\text { les autres }\end{array}$ & $\begin{array}{l}\text { Identifie des } \\
\text { contributeurs }\end{array}$ & $\begin{array}{l}\text { Anonymat } \\
\text { possible }\end{array}$ \\
\hline $\begin{array}{l}\text { Capacité des parties à } \\
\text { remplir leurs obligations }\end{array}$ & $\begin{array}{l}\text { Mieux se } \\
\text { connaitre }\end{array}$ & $\begin{array}{l}\text { Compétences } \\
\text { conceptuelles }\end{array}$ & $\begin{array}{l}\text { Compétences } \\
\text { de prototypage }\end{array}$ & $\begin{array}{l}\text { Compétences } \\
\text { informatiques }\end{array}$ & $\begin{array}{l}\text { Culture } \\
\text { d'entreprise }\end{array}$ & $\begin{array}{l}\text { Critères } \\
\text { d'évaluation } \\
\text { partagés }\end{array}$ & $\begin{array}{l}\text { Partage de la } \\
\text { plus-value }\end{array}$ & $\begin{array}{l}\text { Distribution des } \\
\text { tâches }\end{array}$ \\
\hline $\begin{array}{l}\text { Partage d'objectifs clairs } \\
\text { et appréciés }\end{array}$ & $\begin{array}{l}\text { Décrire le cadre } \\
\text { de travail pour } \\
\text { chacun }\end{array}$ & $\begin{array}{l}\text { Préciser les } \\
\text { modalités de } \\
\text { sélection }\end{array}$ & $\begin{array}{l}\text { Montrer la } \\
\text { progression vers } \\
\text { les objectifs }\end{array}$ & $\begin{array}{l}\text { Exposer et } \\
\text { partager les } 1^{\mathrm{ers}} \\
\text { résultats de la } \\
\text { co-création }\end{array}$ & $\begin{array}{l}\text { Faire se croiser } \\
\text { les } \\
\text { communautés }\end{array}$ & $\begin{array}{l}\text { S'engager sur } \\
\text { des objectifs et } \\
\text { les adapter si } \\
\text { besoin }\end{array}$ & $\begin{array}{l}\text { Être à l'écoute } \\
\text { des besoins }\end{array}$ & $\begin{array}{l}\text { Respecter les } \\
\text { objectifs ou } \\
\text { expliquer les } \\
\text { dérives }\end{array}$ \\
\hline $\begin{array}{l}\text { Environnement non } \\
\text { menaçant Contribuer et } \\
\text { influencer les décisions }\end{array}$ & $\begin{array}{l}\text { Dissiper les } \\
\text { malentendus }\end{array}$ & $\begin{array}{l}\text { Expression de la } \\
\text { divergence }\end{array}$ & $\begin{array}{l}\text { Matérialiser } \\
\text { pour orienter le } \\
\text { choix }\end{array}$ & $\begin{array}{l}\text { Permettre la co- } \\
\text { construction }\end{array}$ & $\begin{array}{l}\text { Encourager le } \\
\text { collectif et } \\
\text { l'esprit d'équipe }\end{array}$ & $\begin{array}{l}\text { Critères } \\
\text { d'impact }\end{array}$ & $\begin{array}{l}\text { Récompenser la } \\
\text { contribution }\end{array}$ & $\begin{array}{l}\text { Garder une } \\
\text { trace et pouvoir } \\
\text { consulter }\end{array}$ \\
\hline $\begin{array}{l}\text { Atteindre l'excellence par } \\
\text { un travail de qualité et } \\
\text { une évaluation critique }\end{array}$ & $\begin{array}{l}\text { Favoriser } \\
\text { l'écoute et } \\
\text { l'échange }\end{array}$ & $\begin{array}{l}\text { Approfondir et } \\
\text { confronter les } \\
\text { idées }\end{array}$ & $\begin{array}{l}\text { Confronter les } \\
\text { idées à leur } \\
\text { faisabilité }\end{array}$ & $\begin{array}{l}\text { Rechercher des } \\
\text { amélioration via } \\
\text { le collectif }\end{array}$ & $\begin{array}{l}\text { Accepter les } \\
\text { critiques } \\
\text { constructives }\end{array}$ & $\begin{array}{l}\text { Confronter les } \\
\text { idées aux } \\
\text { usages }\end{array}$ & $\begin{array}{l}\text { Ethique de la } \\
\text { rétribution }\end{array}$ & $\begin{array}{l}\text { Evaluer l'impact } \\
\text { et termes de } \\
\text { dév. soutenable }\end{array}$ \\
\hline $\begin{array}{l}\text { Valorisation de } \\
\text { l'innovation et soutien } \\
\text { des pratiques }\end{array}$ & $\begin{array}{l}\text { Multiplier les } \\
\text { modes de } \\
\text { communication }\end{array}$ & $\begin{array}{l}\text { Encourager le } \\
\text { dépôt d'idée par } \\
\text { équipe }\end{array}$ & $\begin{array}{l}\text { S'appuyer sur } \\
\text { des espaces } \\
\text { d'innovation }\end{array}$ & $\begin{array}{l}\text { Développer les } \\
\text { smart contrats }\end{array}$ & $\begin{array}{l}\text { Faire } \\
\text { s'approprier le } \\
\text { processus } \\
\text { d'innovation }\end{array}$ & $\begin{array}{l}\text { Partager le } \\
\text { retour } \\
\text { d'expérience }\end{array}$ & $\begin{array}{l}\text { Vers des } \\
\text { propriétés } \\
\text { partagées? }\end{array}$ & $\begin{array}{l}\text { S'inscrire dans la } \\
\text { prospective }\end{array}$ \\
\hline
\end{tabular}

Figure 4. Matrice «co-con » pour renforcer la co-création et la confiance dans un processus d'Innovation Ouvert et Agile

\section{Conclusion et perspectives}

Dans cet article, nous avons rappelé l'aspect fondamental de développer la confiance au sein des processus d'innovation pour garantir et amplifier leur caractère ouvert et renforcer l'agilité. Néanmoins, la littérature souligne le manque de travaux de recherche pour appréhender et caractériser finement les mécanismes sous-jacents à l'établissement d'une confiance partagée dans les processus collaboratifs, tel que la co-création. Deux études de cas basées sur une plus grande prise en compte des usagers dans le domaine de la distribution et de la gestion de l'énergie nous permettent d'illustrer et faire ressortir certains des déterminants de la confiance dans la co-création. Cette étude longitudinale sur des projets qui se sont déroulés entre janvier 2014 et juillet 2017 nécessiterait d'être étayée par l'exploration de nouvelles données issues de travaux spécifiquement orientés sur ce sujet. Par exemple, mettre l'usager ou le consommateur au centre d'un processus d'innovation et au cœur du développement de services ne garantit en rien sa fidélité et son engagement. Sur ce point, chercher à tester la corrélation statistique entre la mise en œuvre de méthodes de co-création et l'appropriation des services ainsi développés par les usagers permettrait de légitimer cette approche. Car comme le mentionne Gassmann et ses collègues [GASSMANN et al., 10], il y a un manque de mesure d'efficacité de ces méthodes. Il serait également pertinent de mener des recherches dans d'autres domaines industriels pour valider la dimension générique du modèle proposé pour conforter le processus d'innovation ouvert et agile (figure 4). Nous n'avons volontairement pas intégré dans ce dernier les technologies du compteur communicant. Celles-ci permettent de recueillir des données d'usage en quasi temps réel ouvrant des perspectives nouvelles dans la relation à l'usager et le développement d'offre de services pour les clients. La création de ces données par les usagers, leur recueil et leur utilisation fait d'ailleurs l'objet d'un débat démocratique en France [ASSEMBLEE NATIONALE, 16]. Les polémiques dans plusieurs pays européens à propos des compteurs communicants soulignent également une certaine méfiance face à ces technologies nouvellement déployées. 
Par ailleurs, la diffusion a priori récente du paradigme d'IO dans le secteur énergétique [GRECO et al., 17] génèrent peut-être des pratiques différentes de secteurs industriels déjà largement engagés dans cette logique. Une approche comparative permettrait sans doute de renforcer le modèle «co-con » que nous proposons. Enfin, Le caractère intangible, voire abstrait, de l'énergie et de l'électricité semble induire une difficulté à mobiliser les acteurs néophytes sur des questions qui peuvent rapidement devenir techniques ou au moins nécessiter le concours de spécialistes pour éclairer des points particuliers. Un plus grand effort de communication, de création de vocabulaire et de représentations partagés semble nécessaire pour pallier cette difficulté. Le développement et l'utilisation d'espaces d'innovation faisant se croiser et collaborer des parties prenantes pourraient apporter un cadre favorable à l'émergence des intelligences collectives [MOREL et al., 18] au service des projets et des écosystèmes. Pour renforcer l'efficience de notre modèle, la dimension temporelle devra également être étudié. En effet, nos deux études de cas décrivent des projets qui se déploient sur des périodes de presque 2 années. Est-ce une constante ou un concours de circonstance? Il conviendrait de mesurer les durées minimales pour assurer le succès de chacune des étapes de la matrice «co-con». Et d'évaluer en quoi le domaine industriel concerné, la diffusion de l'IO, et l'engagement sociétal, influencent la temporalité du processus.

Plus globalement, l'ensemble des éléments de la matrice «co-con» peuvent faire l'objet d'un programme expérimental pour conforter et enrichir cette première proposition d'une meilleure prise en compte de la confiance dans les processus d'innovation ouverte et agile. Par ailleurs, la figure 4 propose des briques de fonctionnalité issues d'une multitude de plates-formes physique et virtuelle. Partant de ce modèle, il serait intéressant d'étudier la possibilité de déployer une technologie numérique intégrant l'ensemble de ces fonctionnalités et qui serait le «jumeau numérique » d'une plate-forme physique dédié à l'innovation collaborative générant un cadre où l'appétence pour la créativité soit véritablement libérée. Où la valeur ajoutée des individus ne serait pas capturée par des « prédateurs » tiers. Où tout un chacun pourrait être un fabricant du futur en harmonie avec ses pairs, dans un climat généralisé d'intelligences collectives. Où des blocs d'inventivité se chaineraient pour former des spirales vertueuses d'intelligences collectives. Où ces spirales créeraient de puissants vortex d'innovation et de disruption... L'émergence récente de la blockchain [NAKAMOTO, 09] support au système de confiance décentralisé semble offrir de nouvelles perspectives dans de très nombreux domaines comme la démocratie [CASEAU, SOUdOPLATOFF, 16] ou encore le manufacturing. Cette technologie semble également apporter des réponses spécifiques aux défis de la co-création [PATRICK DUVAUT et al., 18, SEULLiET, 16]. Des briques technologiques combinées à modèle organisationnel pertinent pourraient dès lors offrir un climat de confiance dans les communautés de co-créateurs et redonner sens et motivation aux acteurs engagés dans des démarches d'innovation ouverte.

\section{Remerciement}

Les auteurs remercient les participants anonymes et volontaires aux projets ELL et LbM. Nous remercions également les institutions, entreprises et espaces d'innovation qui ont soutenu ces travaux s'inscrivant sur du temps long. Laurent Dupont remercie plus particulièrement le Pr. Mauricio Camargo, le Dr. Alex Gabriel et le réseau Daisee pour leur implication dans LbM, leurs conseils et idées. Joëlle Mastelic remercie les entreprises et les particuliers ayant pris part au projet ELL.

\section{Bibliographie}

[Antoniac, Pallot, Pulli, 06] Antoniac, P., Pallot, M., Pulli, P., Virtual and Augmented Reality Supporting Group Consciousness within Collaborative Working Environments, In 2006 IEEE International Technology Management Conference (ICE), IEEE, Milan, Italy, 2006.

[Arnkil, Järvensivu, Koski, PiIrainen, 10] ARnkil, R., Järvensivu, A., Koski, P., PiIRAinen, T., Exploring Quadruple Helix: Outlining user-oriented innovation models. Final Report on Quadruple Helix Research for the CLIQ project, Tampere, 2010. 
[Assemblee Nationale, 16] Assemblee Nationale, Mission d'information commune sur l'application de la loi $n^{\circ}$ 2015-992 du 17 août 2015 relative à la transition énergétique pour la croissance verte, Vol. D, Assemblée Nationale, Paris, France, 2016.

[BAYRAK, 15] BAYRAK, T., « Identifying Collaborative Technology Impact Areas », Technology in Society, 42, p. $93-$ $103,2015$.

[Bente, RÜGgenberg, KrÄMer, 04] Bente, G., RÜGgenBerg, S., KRÄMER, N., Social Presence and Interpersonal Trust in Avatar-Based, Collaborative Net-Communications, In PRESENCE 2004: Proceedings of the 7th Annual International Workshop on Presence, 54-61, 2004.

[CARayannis, Campbell, 12] Carayannis, E. G., CAmPBell, D. F. J., Mode 3 Knowledge Production in Quadruple Helix Innovation Systems, Mode 3 Knowledge Production in Quadruple Helix Innovation Systems, Springer New York, New York, NY, 2012.

[Caseau, Soudoplatoff, 16] Caseau, Y., Soudoplatoff, S., La blockchain ou la confiance distribuée, Fondation pour l'innovation politique, 2016.

[Chesbrough, 03] Chesbrough, H. W., Open Innovation: The New Imperative for Creating and Profiting from Technology, Harvard Business School Press., Boston, 2003.

[Chesbrough, 11] Chesbrough, H. W., « Bringing Open Innovation to Services », MIT Sloan Management Review, 52(2), 2011.

[Cova, Cova, 09] Cova, B., Cova, V., « Les Figures Du Nouveau Consommateur: Une Genèse de La GouverNementalité Du Consommateur », Recherches et Applications En Marketing, 24, p. 1-20, 2009.

[Cruz, Paredes, Fonseca, Morgado, 14] Cruz, A., Paredes, H., Fonseca, B., Morgado, L., « Can Presence Improve Collaboration in 3D Virtual Worlds? », Procedia Technology, 13, p. 47-55, 2014.

[Dikert, PaAsivaara, Lassenius, 16] Dikert, K., PaAsivaAra, M., Lassenius, C., « Challenges and Success Factors for Large-Scale Agile Transformations: A Systematic Literature Review », Journal of Systems and Software, 119, p. 87-108, 2016.

[Dovey, 09] Dovey, K., « The Role of Trust in Innovation », The Learning Organization, 16(4), p. 311-325, 2009.

[Du, Zou, SHI, ZHAO, 18] Du, J., Zou, Z., SHI, Y., ZHAO, D., «Zero Latency: Real-Time Synchronization of BIM Data in Virtual Reality for Collaborative Decision-Making », Automation in Construction, 85(October 2017), p. 51-64, 2018.

[Dupont, Gabriel, Camargo, Guidat, 17] Dupont, L., Gabriel, A., CAMArgo, M., Guidat, C., Collaborative Innovation Projects Engaging Open Communities: a Case Study on Emerging Challenges., In 2017 International Conference on Engineering, Technology and Innovation (ICE/ITMC), June 27-29, 1122-1131, IEEE Technology and Engineering Management Society (TEMS), Funchal, Madeira Island, Portugal, 2017.

[Dupont, Gholipour, Morel, Bignon, Guidat, 12] Dupont, L., Gholipour, V., Morel, L., Bignon, J.-C., Guidat, C., « From Urban Concept to Urban Engineering: The Contribution of Distributed Collaborative Design to the Management of Urban Projects », Journal of Urban Design, 17(2), p. 255-277, 2012.

[Dupont, Morel, Guidat, 15] Dupont, L., Morel, L., Guidat, C., « Innovative Public-Private Partnership to Support Smart City: The Case of "Chaire REVES" », Journal of Strategy and Management, 8(3), p. 245-265, 2015.

[Dupont, Morel, Guidat, Hubert, Revel, 15] Dupont, L., Morel, L., Guidat, C., Hubert, J., Revel, M., « Le «technicien-Citoyen » et La Fabrique Nancy Grand Cœur: Le Prisme de l'usage Dans La Conception d'un Écoquartier », In Mermet, L., Salles, D. (Dir.) "Environnement: la concertation apprivoisée, contestée, dépassée ?, ”233-256, De Boeck, Louvain-La-Neuve, 2015. Retrieved from http://www.deboecksuperieur.com/titres/132570_3/9782804191085-environnement-la-concertation-apprivoiseecontestee-depassee.html

[Dupont, Morel, Hubert, Guidat, 14] Dupont, L., Morel, L., Hubert, J., Guidat, C., Study case: Living Lab Mode for urban project design: Emergence of an ad hoc methodology through collaborative innovation, In 2014 International Conference on Engineering, Technology and Innovation (ICE), 1-9, IEEE, Bergamo, Italy, 2014.

[Dupont, Morel, Lhoste, 15] Dupont, L., Morel, L., Lhoste, P., Le Lorraine Fab Living Lab : la 4ème dimension de l'innovation, In Actes des sessions du colloque Science \& You, France, 230-235, Université de Lorraine, Nancy, France, 2015. Retrieved from http://www.science-and-you.com/sites/science-andyou.com/files/users/sy2015_sessions_proceedings.pdf

[Dupont, Pallot, Christmann, Richir, 18] Dupont, L., Pallot, M., Christmann, O., Richir, S., A Universal Framework For Systemizing the Evaluation of Immersive And Collaborative Performance, In ACM VRIC'18 Laval Virtual, ACM, Laval, France, 2018. 
[Eynard, Pallot, Christmann, RichiR, 15] Eynard, R., PAllot, M., Christmann, O., Richir, S., Impact of Verbal Communication on User Experience in 3D Immersive Virtual Environments, In IProceedings of the International Conference on Engineering, Technology and Innovation (ICE), ICE'2015, Belfast, Northern Ireland, 2015.

[Fawcett, Jones, Fawcett, 12] Fawcett, S. E., Jones, S. L., Fawcett, A. M., « Supply Chain Trust: The Catalyst for Collaborative Innovation », Business Horizons, 55(2), p. 163-178, 2012.

[FREINA, OTT, 15] FREINA, L., OTT, M., A literature review on immersive virtual reality in education: State of the art and perspectives, In Proceedings of eLearning and Software for Education (eLSE)(Bucharest, Romania, April 23-24, 2015), 8, Bucharest, Romania, 2015.

[Fu, Hwang, 18] Fu, Q.-K., Hwang, G.-J., « Trends in Mobile Technology-Supported Collaborative Learning: A Systematic Review of Journal Publications from 2007 to $2016 »$, Computers \& Education, 119(December 2017), p. 129-143, 2018.

[Gábana Arellano, TokarchuK, Gunes, 16] Gábana Arellano, D., ToKarchuK, L., Gunes, H., Measuring affective, physiological and behavioural differences in solo, competitive and collaborative games, In 8th International Conference on Intelligent Technologies for Interactive Entertainment - Intertain (June, 28-30), 1-10, Utrecht, Netherlands, 2016.

[Gassmann, Enkel, Chesbrough, 10] Gassmann, O., Enkel, E., Chesbrough, H., « The Future of Open Innovation », R\&D Management, 40(3), 2010.

[GReco, Locatelli, Lisi, 17] Greco, M., Locatelli, G., Lisi, S., « Open Innovation in the Power \& Energy Sector: Bringing Together Government Policies, Companies’ Interests, and Academic Essence », Energy Policy, 104(February), p. 316-324, 2017.

[Guidat, Dupont, Skiba, Bretagne, Camargo, Massouras, 11] Guidat, C., Dupont, L., Skiba, N., Bretagne, V., CAMARGO, M., MASSOURAS, G., Lorraine Smart Cities Living Lab: white paper on living labs (Livre blanc remis à la DIRECCTE), Institut National Polytechnique de Lorraine (INPL), Nancy, France, 2011.

[Highsmith, Fowler, 01] Highsmith, J., Fowler, M., « The Agile Manifesto », Software Development Magazine, 9(8), p. 29-30, 2001.

[Jennett, Cox, Cairns, Dhoparee, Epps, Tijs, Walton, 08] Jennett, C., CoX, A. L., Cairns, P., Dhoparee, S., EPPS, A., TIJS, T., WAlton, A., « Measuring and Defining the Experience of Immersion in Games », International Journal of Human Computer Studies, 66(9), p. 641-661, 2008.

[Ke, CARAFAnO, 16] Ke, F., CARAFAnO, P., «Collaborative Science Learning in an Immersive Flight Simulation », Computers and Education, 103, p. 114-123, 2016.

[Koirala, Araghi, Kroesen, Ghorbani, Hakvoort, Herder, 18] Koirala, B. P., Araghi, Y., Kroesen, M., GHORBANI, A., HAKVOORT, R. A., HERDER, P. M., « Trust, Awareness, and Independence: Insights from a SocioPsychological Factor Analysis of Citizen Knowledge and Participation in Community Energy Systems », Energy Research and Social Science, 38(December 2016), p. 33-40, 2018.

[Le, Fueld, Alavi, KunZ, 17] Le, K.-D., FJeld, M., Alavi, A., KunZ, A., « Immersive Environment for Distributed Creative Collaboration », Proceedings of the 23rd ACM Symposium on Virtual Reality Software and Technology VRST'17, p. 1-4, 2017.

[LI, Yi, ChI, WANG, Chan, 18] Li, X., YI, W., ChI, H. L., WANG, X., Chan, A. P. C., « A Critical Review of Virtual and Augmented Reality (VR/AR) Applications in Construction Safety », Automation in Construction, 86(October 2017), p. 150-162, 2018.

[Lorenzo, Ángel Sicilia, SÁnchez, 12] Lorenzo, C. M., Ángel Sicilia, M., SÁnchez, S., « Studying the Effectiveness of Multi-User Immersive Environments for Collaborative Evaluation Tasks », Computers and Education, 59(4), p. 1361-1376, 2012.

[Louchart, Aylett, Hall, Woods, Paiva, 06] Louchart, S., Aylett, R., Hall, L., Woods, S., Paiva, A., FearNot! Developing Social Immersion in the VICTEC and ECIRCUS Projects, In Ludovia 2006 Université du multimédia ludo-éducatif et pédagogique, Saint Lizier, France, 2006.

[MAYER, DAVIS, SchOORMAN, 95] MAYeR, R. C., DAVIS, J. H., SCHOORMAN, F. D., « An Integrative Model of Organizational Trust. », Academy of Management Review, 20(3), p. 709-734, 1995.

[Mergel, 16] Mergel, I., « Agile Innovation Management in Government: A Research Agenda », Government Information Quarterly, 33(3), p. 516-523, 2016.

[Morel, Dupont, Boudarel, 18] Morel, L., Dupont, L., Boudarel, M.-R., Espace d'innovation : de nouveaux lieux pour l'intelligence collective?, 2018. 
[NAKAмото, 09] NAKAмото, S., « Bitcoin: A Peer-to-Peer Electronic Cash System », 2009.

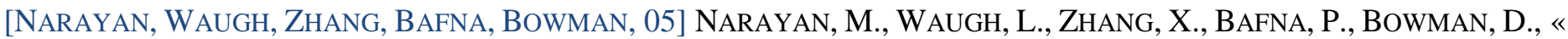
Quantifying the Benefits of Immersion for Collaboration in Virtual Environments », Proceedings of the ACM Symposium on Virtual Reality Software and Technology - VRST '05, p. 78, 2005.

[Orenstein, Zimroni, EizenberG, 15] Orenstein, D. E., Zimroni, H., EizenberG, E., « The Immersive Visualization Theater: A New Tool for Ecosystem Assessment and Landscape Planning », Computers, Environment and Urban Systems, 54, p. 347-355, 2015.

[Pallot, 12] Pallot, M., Collaborative Distance: Investigating Distance Factors Affecting Collaboration Performance, LAP LAMBERT Academic Publishing, 2012.

[PAllot, Richir, 16] PALlot, M., RichiR, S., Laval Virtual Vision 2025 : Blurring the lines between digital and physical worlds, In Proc. 11th Intl Conf. Disability, Virtual Reality \& Associated Technologies, 1-9, ICDVRAT, Los Angeles, USA, 2016.

[Patrick Duvaut, Seulliet, Shavit, 18] Patrick Duvaut, Seulliet, E., Shavit, D., « La Blockchain Pour Redonner Le Pouvoir Aux Individus », (R. Heinz, Ed.) Harvard Business Review France, PRISMA MEDIA, Paris, 2018 , January 20. Retrieved from https://www.hbrfrance.fr/chroniques-experts/2018/01/18712-reinventercocreation-grace-a-blockchain/

[Pirola-Merlo, 10] Pirola-Merlo, A., « Agile Innovation: The Role of Team Climate in Rapid Research and Development », Journal of Occupational and Organizational Psychology, 83(4), p. 1075-1084, 2010.

[Pouliquen-Lardy, 16] Pouliquen-LaRdy, L., Collaboration à distance : étude de la compréhension mutuelle dans les environnements virtuels collaboratifs immersifs - Le cas de lacommunication spatiale, UNIVERSITE RENNES 2, 2016 , May.

[Recker, Mendling, Hahn, 13] Recker, J., Mendling, J., Hahn, C., « How Collaborative Technology Supports Cognitive Processes in Collaborative Process Modeling: A Capabilities-Gains-Outcome Model », Information Systems, 38(8), p. 1031-1045, 2013.

[Roth, 15] Roth, C., Experiencing Interactive Storytelling, Vrije University, Amsterdam, 2015.

[SAntos, Soares, Carvalho, Raposo, 12] Santos, I. H. F., Soares, L. P., Carvalho, F., Raposo, A., « A Collaborative Virtual Reality Oil \& Gas Workflow. », The International Journal of Virtual Reality, 11(1), p. 1-13, 2012.

[Seulliet, 16] Seulliet, E., « Open Innovation, Co-Création: Pourquoi La Blockchain Est Une Petite Révolution », Harvard Business Review France, Chroniques(HBR France Website), p. 5-9, 2016.

[ShaZi, Gillespie, SteEn, 15] ShAZI, R., GillesPie, N., SteEn, J., « Trust as a Predictor of Innovation Network Ties in Project Teams », International Journal of Project Management, 33(1), p. 81-91, 2015.

[ŞTEFAN, 12] ŞTEFAN, L., « Immersive Collaborative Environments for Teaching and Learning Traditional Design », Procedia - Social and Behavioral Sciences, 51, p. 1056-1060, 2012.

[TRAN, 14] TRAN, S., Quelle contribution des technologies collaboratives à la configuration des organisations?, Systèmes d'information \& management, Vol. 19, 2014.

[Ulewicz, PANTFÖRder, Vogel-Heuser, 16] Ulewicz, S., PANTFÖRder, D., Vogel-Heuser, B., « Interdisciplinary Communication and Comprehension in Factory Automation Engineering - A Concept for an Immersive Virtual Environment », IFAC-PapersOnLine, 49(19), p. 227-232, 2016.

[VAn Der Valk, Sumo, Dul, Schroeder, 16] Van Der Valk, W., Sumo, R., Dul, J., Schroeder, R. G., « When Are Contracts and Trust Necessary for Innovation in Buyer-Supplier Relationships? A Necessary Condition Analysis », Journal of Purchasing and Supply Management, 22(4), p. 266-277, 2016.

[Wang, Yeung, Zhang, 11] Wang, L., Yeung, J. H. Y., Zhang, M., « The Impact of Trust and Contract on Innovation Performance: The Moderating Role of Environmental Uncertainty », International Journal of Production Economics, 134(1), p. 114-122, 2011.

[West, 90] West, M. A., «The Social Psychology of Innovation in Groups », In M. A. WeST and J. L. FARR (Eds.), Innovation and creativity at work: Psychological and organizational strategies, 101-122, Wiley, New York, 1990.

[West, Sacramento, 06] West, M. A., Sacramento, C. A., « Flourishing in Teams: Developing Creativity and Innovation », Creative Management and Development, Third Edition, p. 25-44, 2006.

[YIN, 13] YIn, R. K., Case Study Research: Design and Methods, 5th ed., SAGE Publications, 2013. Retrieved from https://books.google.fr/books?id=OgyqBAAAQBAJ 